
\title{
25 Research Square \\ Calcium Binding Protein S100A16 Expedites Proliferation, Invasion and EMT Process in Gastric Cancer
}

\section{Xiaoying You}

Nanjing Medical University

Min Li

Nanjing Medical University

Hongwei Cai

Nanjing Medical University

Wenwen Zhang

Children's Hospital of Nanjing Medical University

Ye Hong

Nanjing Medical University

Wenjie Gao

Nanjing Medical University Second Affiliated Hospital

Yun Liu

Jiangsu Province Hospital and Nanjing Medical University First Affiliated Hospital

Xiubin Liang

Nanjing Medical University

TIJUN WU ( $\nabla$ wutijun@njmu.edu.cn )

Nanjing Medical University https://orcid.org/0000-0002-8788-5686

\section{Fang Chen}

Nanjing Medical University

Dongming Su

Nanjing Medical University

\section{Research}

Keywords: S100A16, gastric cancer, metastasis, tight junctions, ZO-2

Posted Date: July 9th, 2021

DOI: https://doi.org/10.21203/rs.3.rs-674611/v1 
License: (c) (i) This work is licensed under a Creative Commons Attribution 4.0 International License. Read Full License 


\section{Abstract}

Background: Gastric cancer (GC) is one of the most common malignant tumors of the digestive system, which has been the second cause of cancer-related deaths worldwide. The distant metastasis is one of the main reasons for the high recurrence and mortality rate of GC patients. Hence, it is necessary to investigate the molecular mechanism underlying gastric carcinogenesis and progression, especially the key genes and signaling pathways that promote GC cells proliferation, invasion, and metastasis.

Methods: Using bioinformatics and clinicopathological analysis, in vivo tumor formation assays, mass spectrometry and so on, we characterized the role and molecular mechanism of S100 Calcium Binding Protein A16 (S100A16) in promoting GC tumor growth, migration, invasion and epithelial-to-mesenchymal transition (EMT), and investigated how Zonula Occludens-2 (ZO-2) inhibition mediates S100A16-induced metastasis and progression in GC.

Results: We analyzed S100A16 expression with the GEPIA database and the UALCAN cancer database, and the prognostic analysis was performed using 100 clinical GC samples. We found that S100A16 is significantly upregulated in GC tissues and closely correlated with poor prognosis in GC patients. Functional studies reveal that S100A16 overexpression triggers $\mathrm{GC}$ cells proliferation and migration both in vivo and in vitro; by contrast, S100A16 knockdown restricts the speed of GC cells growth and mobility. Proteomic analysis results reveal a large S100A16 interactome, which includes ZO-2, a master regulator of cell-to-cell tight junctions. Mechanistic assay results indicate that excessive S100A16 instigates GC cell invasion, migration and EMT via ZO-2 inhibition, which arose from S100A16-mediated ZO-2 ubiquitination and degradation.

Conclusions: Our results not only reveal that S100A16 is a promising candidate biomarker in GC early diagnosis and prediction of metastasis, but also establish the therapeutic importance of targeting S100A16 in order to prevent ZO-2 loss and suppress GC metastasis and progression.

\section{Introduction}

Gastric cancer (GC) is the fourth most commonly diagnosed cancer and the second leading cause of cancer-related deaths worldwide [1]. Globally, there are an average of 989,600 new cases and 738,000 GC mortalities occurs every year [1-3]. Various risk factors are positively correlated with this fatal malignant tumor, including environmental factors, genetic instability, genetic factor defects, bacterial factors, i.e., Helicobacter pylori infection and host-related factors [4]. Although early GC screening improves overall survival, the prognosis of GC is still poor when compared with other solid tumors [5], since a majority of GC patients are still diagnosed with advanced stage, accompanied by regional, distant, or both, metastasis [6]. The treatment for GC patients is mainly radical surgical resection and chemoradiotherapy, while metastatic patients are less effective in surgical treatment, more resistant to drug therapy, and thus have lower survival rates [7-9]. Therefore, it is necessary to investigate the molecular mechanism 
underlying gastric carcinogenesis and progression, especially the key genes and signaling pathways that promote GC cells proliferation, invasion, and metastasis.

In human, dysregulated expression patterns of S100 proteins family is a common feature of tumor progression, of each type exhibits unique $\mathrm{S} 100$ protein profile or characteristics [10]. S100 family is the largest subfamily of calcium $\left(\mathrm{Ca}^{2+}\right)$-binding proteins which is composed of 22 components that act as intracellular $\mathrm{Ca}^{2+}$ sensors and extracellular factors to regulate cellular responses [11, 12]. It has been widely indicated that $\mathrm{S} 100$ members participate in multiple stages of tumorigenic process such as cell proliferation, tumor invasion, angiogenesis and immune evasion [10]. Notably, S100 proteins interact with receptor for advanced glycation end-products (RAGE), p53 and p21, which play a role in the degradation of the extracellular matrix (ECM) and metastasis [13]. S100 Calcium Binding Protein A16 (S100A16), the most recent member of $\mathrm{S} 100$ family proteins, functions to increase the tumor progression. The molecular mechanisms of S100A16 involving in the tumor metastasis are diverse in various malignant tumors, including pancreatic cancer [14, 15], leukemia [16], breast cancer [17], and so on. In gastric cancer, decreased S100A16 expression is involved in miR-6884-5p and ADAMTS19 (A Disintegrin and Metalloproteinase with Thrombospondin motifs 19) inhibition of GC cells migration, invasion, and EMT $[18,19]$, however, the role of S100A16 itself in GC metastasis have not been fully clarified yet.

Tight junctions (TJs), as the topmost structure between epithelial cells and endothelial cells, is recognized as the control element for the diffusion of ions and certain molecules around the cell. It is becoming increasingly obvious that TJ plays a vital role in maintaining the integrity of cells, and the loss of cohesion of its structure can lead to the invasion and metastasis of cancer cells [20-22]. Zonula occludens proteins ZO-2 belongs to the membrane associated guanylate kinase homologue (MAGUK) protein family and is concentrated at the cytoplasmic face of TJs in epithelial cells [23]. Based on recent research, the interaction of ZO-2 with viral oncoproteins and kinases and its silencing in diverse carcinomas reinforce the view of ZO-2 as a tumor regulator protein [24-27]. In line with this, ZO-2 absence is also responsible for the diffusely infiltrating growth and frequent metastatic spread of GC cells $[28,29]$, while the specific molecular mechanism remains unclear.

In the current study, we demonstrated that S100A16 is considerably induced to trigger GC metastasis and progression via downregulating ZO-2 levels. We observed that S100A16 expression is dramatically increased in gastric cancer tissues compared with adjacent normal tissues, and S100A16 elevation is a significant prerequisite for GC proliferation, invasion and migration. Using mass spectrometric analysis, we identified a large group of potential interactome for S100A16 in GC cells, including Zonula occludens proteins ZO-2 which acts as one core element in tight junctions. Moreover, we found that S100A16 is responsible for ZO-2 ubiquitination and degradation, resulting in the reduced cellular content of ZO-2, leading to enhanced spread of GC metastasis.

\section{Materials And Methods}

\section{Cell culture and treatment}


The non-malignant gastric mucosal epithelial cell line GES-1 (RRID: CVCL_EQ22) and human GC cell lines (MGC-803, RRID: CVCL_5334; SGC-7901, RRID: CVCL_0520) were purchased from ATCC. Cells were cultured in RPMI-1640 medium (Gibco, USA) with 10\% fetal bovine serum (Wisent, China). The cells were cultured at $37^{\circ} \mathrm{C}$ in an incubator supplemented with $5 \% \mathrm{CO}_{2}$.

\section{Gene overexpression and knockdown}

Human lentivirus-S100A16, adenovirus-S100A16, lentivirus-ZO-2 and their control virus were purchased from Genechem (Shanghai, China). SGC-7901 and MGC-803 cell lines that stably overexpressing S100A16 and/or ZO-2 were established via being infected with lentivirus-S100A16 and/or ZO-2 as described previously [30]. Specific siRNA for S100A16 (si-S100A16) was purchased from GenePharma (Shanghai, China). Transient transfection with interfering RNA (siRNA) and plasmids was performed with Lipofectamine 2000 (Invitrogen) according to manufacturer's protocol.

\section{Patients and tissue specimens}

The initial 8 cases of gastric cancer samples from patients were obtained as FFPE (formaldehyde-fixed and paraffin-embedded) tissues from the Department of Pathology in Sir Run-Run Hospital of Nanjing Medical University. The tissue microarray was purchased from Shanghai Xinchao Biotechnology Co., Ltd. It contains 100 cases of gastric cancer and 80 cases of adjacent tissue sites, of which 80 cases are matched specimens.

\section{In vivo tumor formation}

All procedures were approved by the Institutional Animal Care and Use Committee of Nanjing Medical University. 6-week-old male BALB/C nude mice were purchased from CAVENS LAB ANIMAL (China). For in vivo tumor formation, SGC-7901 cells, either stably overexpressing S100A16 or its control lentivirus, were resuspended in 1:1 PBS/Matrigel (356,237, Corning) solution and injected subcutaneously in the flank of nude mice at a density of $5 \times 10^{6}$ cells in $0.1 \mathrm{ml}$ per mouse. Mice were kept in a specific-pathogen-free (SPF) environment and tumor size was measured weekly since $5 \mathrm{~d}$ after injection. Four weeks after injection, euthanasia was administered, and tumors were isolated with the volume calculated according to the formula Volume $=\left(\right.$ long diameter $\mathrm{x}$ short diameter $\left.{ }^{2}\right) / 2$.

\section{Immunohistochemistry staining (IHC)}

All protocols for animal experimentation and maintenance adhered to the guidelines of the Institutional Animal Care and Use Committee at Nanjing Medical University. IHC was performed as previously described [30]. Briefly, tissues were deparaffinized and rehydrated firstly, then blocked with 5\% BSA in PBS at room temperature for 30 minutes. Afterward, slides were incubated overnight at $4^{\circ} \mathrm{C}$ with antibodies against S100A16 (Sigma) and ZO-2 (Abcam) in blocking buffer. After being washed, the specimens were incubated in HRP-conjugated secondary antibody and visualized using a DAB Peroxidase Substrate Kit 
(Gene Tech). Images were obtained using a laser scanning microscope (IX-51, Olympus), and IHC scores were evaluated by three independent pathologists.

\section{Western blotting and immunoprecipitation}

The total proteins of lysates from human GC cell lines (MGC-803 and SGC-7901) and GES-1 cell line were separated by $8 \%$ or $12 \%$ SDS-PAGE which containing $1.5 \mathrm{~mol} / / \mathrm{I}$ Tris- $\mathrm{HCl}(\mathrm{pH} 8.8), 1 \mathrm{~mol} / \mathrm{I} \mathrm{Tris-} \mathrm{HCl}(\mathrm{pH} 6.8)$, $30 \%$ Acr-Bis, $10 \%$ APS, $10 \%$ SDS and TEMED. $5 \%$ non-fat milk was used to block the unbound sites for 2 hours at room temperature. The protein was blotted with primary antibodies against S100A16 (Proteintech Group), ZO-2 (Proteintech Group), E-Cadherin (Cell Signaling Technology), Twist (Cell Signaling Technology), Vimentin (Cell Signaling Technology) and then were incubated with secondary antibodies. GAPDH was detected as a loading control. For immunoprecipitation, whole cell proteins were extracted using NP40 lysis, the pull-down assay was performed as we described previously [30].

\section{RNA isolation and quantitative RT-PCR analysis}

Total RNA was extracted by TRIzol ${ }^{\text {TM }}$ Plus RNA Purification Kit (Invitrogen, USA) according to the manufacturer's instructions. cDNA was prepared from RNA using the ReverTra Ace RT-PCR Kit (TOYOBO Biotech, Japan). All the RT-PCR reactions were performed with SYBR Green Master (Roche Molecular Systems, Switzerland). $\beta$-actin was used as an internal control.

\section{Cell immunofluorescence staining}

For Immunofluorescence staining, the SGC-7901 cells and MGC-803 cells were fixed in 4\% paraformaldehyde overnight at $4{ }^{\circ} \mathrm{C}$, followed by three washes with PBS carefully to remove any debris. After fixation, $0.2 \%$ Triton X-100 was used for cell membrane permeabilization. 15 minutes later, cells were blocked by $1 \%$ BSA at room temperature for $1 \mathrm{~h}$ and incubated overnight at $4{ }^{\circ} \mathrm{C}$ with anti-S100A16 and anti-ZO-2 antibodies. The next day cells were washed with PBS for three times, then the cells were incubated with appropriate secondary antibodies for 30 minutes at $37^{\circ} \mathrm{C}$. The cells were stained with $4,6-$ diamidino-2-phenylindole for 2 minutes at room temperature and then washed three times with PBS. All images were obtained from Olympus confocal microscope and processed using Photoshop software.

\section{Cell proliferation and colony formation assays}

For the cell proliferation assay, $24 \mathrm{~h}$ after transfection, tumor cells were seeded into 96 -well plates at a density of 1500 per well and cultured for 5 days. Each well was added with $100 \mu \mathrm{l} \%$ TCA for fixation overnight at $4{ }^{\circ} \mathrm{C}$ daily. After being washed with $\mathrm{ddH}_{2} \mathrm{O}$ for 3-5 times, these plates were put into oven until no moisture remains. Each well was then added $50 \mu \mathrm{l}$ Sulforhodamine B (SRB) for 30 minutes at room temperature and then washed with $1 \%$ acetic acid solution for 5 times, and this procedure was repeated. Finally, $100 \mu \mathrm{l} 10 \mathrm{nmol} / \mathrm{I}$ Tris ( $\mathrm{pH}=10.5)$ was added into each plate to dissolve the SRB. The optical density at $490 \mathrm{~nm}$ (OD490), in linear correlation with the number of living cells, was measured by Varioskan ${ }^{\mathrm{TM}}$ LUX microplate reader (Thermo Fisher Scientific). For the colony formation assay, tumor cells 
(500 cells per well) were seeded into 6-well plates. After 2 or 3 weeks of incubation, the cells were fixed in $4 \%$ paraformaldehyde and then stained with crystal violet for 30 minutes (Beyotime Biotechnology, China). The colonies were counted via Image J (version 1.8.0).

\section{Cell invasion Assays}

Cell migration ability was measured using transwell chambers $(8 \mu \mathrm{m}$ pore size; Corning Costar, Cambridge, MA, USA). For the transwell assay, cells cultured in serum-free RPMI- 1640 medium were seeded into the upper chamber. The lower chamber contained RPMI-1640 medium supplemented with $20 \%$ serum, which served as a chemoattractant. After 24 or $48 \mathrm{~h}$ incubation, the filters were fixed in $4 \%$ paraformaldehyde for $1 \mathrm{~h}$ and stained with crystal violet. The upper faces of the filters were gently removed, and the lower faces with cells migrated across the filters were imaged and counted under the microscope (IX-51, Olympus).

\section{Mass spectrometry analysis}

The mass spectrometry was performed on the precipitated protein of SGC-7901 cells transfected with Lentivirus-S100A16 or empty vectors. The labeled peptides were analyzed on the LTQ-Orbitrap instrument (Thermo Fisher, USA) connecting to a Nano ACQUITY UPLC system via a nanospray source. The LC$\mathrm{MS} / \mathrm{MS}$ was operated in positive ion mode. The analytical condition was set at a linear gradient from 0 to $60 \%$ of buffer $\mathrm{B}(\mathrm{CH} 3 \mathrm{CN})$ in $150 \mathrm{~min}$, and flow rate of $200 \mathrm{nl} / \mathrm{min}$. For analysis of proteins from MIN6 cells, one full MS/MS scan was followed by five MS/MS scans on those five highest peaks respectively. The MS/MS spectra acquired from precursor ions were submitted to Maxquant (version 1.2.2.5) using the following search parameters: the database searched was Uniprot proteome (version20120418); the enzyme was trypsin (KR/P); the dynamic modifications were set for oxidized Met (+16); carbamidomethylation of cysteine was set as static modification; MS/MS tolerance was set at 10ppm; the minimum peptide length was 6 ; the false detection rate for peptides, proteins were all set below 0.01 [31].

\section{Wound closure assay}

Cells were plated in 3.5-cm dishes and were divided into 12-well plates to achieve $70-80 \%$ confluence and then wounded by dragging a plastic pipette tip across the monolayer surface three times, followed by gentle washing with PBS twice. Images of the wounds were recorded with a Leica DM IRB inverted microscope (Solms, Germany).

\section{Measurement of MMP-2, MMP-9 and TIMP-1 levels}

The supernatants of SGC-7901 and MGC-803 cells were collected after indicated treatment, and the concentrations of matrix metalloproteinase-2 (MMP-2), MMP-9, and tissue inhibitor of metalloproteinase-

1 (TIMP-2) were measured by ELISA kit (USCN Life Science, China) according to the manufacturer's instructions. 


\section{CHX chase assay}

SGC-7901 cells infected with lenti-S100A16 or lenti-control were seeded in 6-well plates at a density of 1 $\times 10^{5}$ cells per well and cultured overnight. Then, cells were treated with $50 \mu \mathrm{g} / \mathrm{ml}$ of cycloheximide (CHX) and harvested after $0 / 12 / 24 / 48 \mathrm{~h}$ of $\mathrm{CHX}$ treatment. The total protein was extracted and subjected to SDS-PAGE to detect the protein level of ZO-2 at different time points, indicating ZO-2 stability in SGC7901 cells overexpressing S100A16.

\section{Plasmid construction}

hZO-2-Flag, hZO-2AU2 region-Flag, hS100A16-Myc were generated using a QuikChange Site-Directed Mutagenesis kit (Stratagene, La Jolla, California) according to manufacturer's instructions.

\section{Cell-based ubiquitination assay}

HEK293A cells were transfected with plasmids encoding hZO-2-Flag or hZO-2AU2 region-Flag, hS100A16Myc, and Ubiquitin-HA. After transfection for $24 \mathrm{~h}$, the cells were incubated with the proteasome inhibitor MG132 (Sigma Aldrich) for $4 \mathrm{~h}$ and then lysed. Equal amounts of total cell lysates were incubated with the Flag antibodies (Sigma) overnight at $2{ }^{\circ} \mathrm{C}$. Immunocomplexes were collected overnight at $4{ }^{\circ} \mathrm{C}$ using protein-A sepharose beads (Roche). The immunoprecipitates were washed with lysis buffer and subjected to Western blot analysis with anti-HA antibody (Cell Signaling Technology).

\section{Statistical analysis}

GraphPad Prism 8.0.1 (GraphPad Software, Inc. La Jolla, CA, USA) and Microsoft Excel 2019 were used for statistical analysis and the data were presented as mean \pm standard deviation. Data were statistically analyzed using one-way ANOVA with a Bonferroni correction, followed by Fisher's exact test for comparison of two groups.

\section{Results}

\section{S100A16 is elevated in GC tissues and correlated with poor prognosis in GC patients}

It has been widely reported before that S100A16 is upregulated in various type of cancer cells in the comparison with normal tissues. To further determine the role of S100A16 in progression of gastric cancer (GC), we analyzed S100A16 expression in GC tissues with GEPIA (http://gepia.cancer-pku.cn/) and UALCAN (http://ualcan.path.uab.edu) databases. S100A16 was significantly more excessive in GC tissues when compared with normal tissues (Figure 1A-B). Based on this, we utilized UALCAN database to explore clinic pathological features of S100A16 in GC samples. As shown in Figure 1C-D, S100A16 expression was positively correlated with $H$. pylori infection (a recognized GC pathogenic factor), as well as with the tumor grade of GC, which further indicated that S100A16 is highly possible to serve as a considerable diagnostic and prognostic indicator in GC. 
We then confirmed that database analysis mentioned above. Using gastroscopic biopsy tissues obtained from GC patients, we demonstrated that S100A16 expression in GC tissues was dramatically higher than that in adjacent normal tissues (Figure 2A-B). Similarly, elevated S100A16 was also observed in GC tumors when compared with adjacent normal tissues according to tissue microarray (patient clinicopathological characteristics shown in Table 1), whether it was matched or not (Figure 2C-D). We further studied the relationship between S100A16 and clinical GC pathology. According to the IHC score

for S100A16 (Table 2), all GC patients were divided into 2 groups: S100A16 high (IHC score $\geq 2,25$ cases) and S100A16 ${ }^{\text {low }}$ (IHC score $<2,72$ cases). Kaplan-Meier analysis revealed that S100A16 $6^{\text {high }}$ patients had an obvious worse prognosis than those in low expression groups (Figure 2E), and the results above were still statistically significant (Figure 2F, $p<0.00043$ ) after adjustment for other factors (such as gender, pathological grade, tumor size, and Ki67 index, shown in Table 3). Therefore, both bioinformatics and clinicopathological analysis prove that S100A16 is highly expressed in GC, which play roles in GC progression.

\section{Overexpression of S100A16 triggers GC cells growth and migration in vitro and in vivo}

In accordance with $\mathrm{IHC}$ results in Figure 2, we observed elevated S100A16 mRNA and protein levels in human GC cell lines SGC-803 and SGC-7901 when compared with gastric mucosa cell line GES-1 (Figure 3A-C). We next applied those two cell lines to investigate the biological function of S100A16 in GC cells. As shown in Figure 3D, S100A16 stably overexpression promoted the growth rate of SGC-7901. Colony formation assays further indicated that S100A16 overexpression enhances the capacity of SGC-7901 proliferation (Figure 3E-F). The pro-proliferation effect of S100A16 were also observed in MGC-803 cells (Figure 3G-I). Besides, migration capacities were assessed to be notably increased in SGC-7901 and MGC803 overexpressing S100A16 by transwell assays (Figure 3J-M). To further evaluate the effect of S100A16 on GC cell migration and invasion in vivo, we constructed subcutaneous tumor formation experiments in nude mice using SGC-7901 cells stably infected with lenti-S100A16 or scramble control (lenti-S10016 or control group, Figure 4A). S100A16 overexpression in transplanted GC tumor tissues were confirmed in Figure 4B-C via Western blotting and IHC. The growth rate of tumor grafts in the mice from lenti-S100A16 group was significantly faster than that in control-group from the 10th day of SGC7901 infection (Figure 4D). We then sacrificed those mice and extracted the transplanted tumor tissues for observation. As we expected, the size and weight of tumor grafts isolated from lenti-S100A16 group were obviously larger than that in control group after 26 days of tumor implantation (Figure 4E-F). Collectively, S100A16 promotes GC cells migration, proliferation, and growth.

\section{S100A16 inhibition suppresses GC cells growth and migration}

We applied loss-of-function experiments to further determine the role of S100A16 in GC cells proliferation and migration. As shown in Figure 5A-D, S100A16 knock-down restricted the growth rate and colony formation of SGC-7901 cells. Also, the similar results were verified in MGC-803 cells (Figure 5F-H). Furthermore, transwell assay results also demonstrated that S100A16 knockdown suppressed the migration of MGC-803 and SGC-7901 cells (Figure 5I-L). Together, S100A16 upregulation is both 
necessary and sufficient to trigger GC tumor growth, proliferation, and migration, which suggest that it functions as a considerable oncogene in GC.

\section{S100A16 and ZO-2 interact directly and their expression levels are inversely correlated in GC cells}

To determine the molecular basis of the increased proliferation and migration in GC cells with high S100A16 expression, we performed a mass spectrometric analysis to investigate the binding proteins of S100A16 in lenti-S100A16- or lenti-scramble-infected SGC-7901 cells. Data showed that 67 proteins interacted with S100A16, among which 25 proteins showed weakened (8 proteins) or stronger (17 proteins) affinity with S100A16 when S100A16 was highly expressed in SGC-7901 cells (Table 4). Importantly, GO analysis with DAVID Bioinformatics Resources 6.7 (Figure 6A) showed that 3 of those 25 S100A16-interacting proteins were involved in cell-to-cell adherens junction which may be associated with tumor cell migration and invasion. Among these proteins is TJP2 (also called ZO-2), which is an important regulator in tumor growth and metastasis (Figure 6B-C). Immunoprecipitation analyses confirmed that endogenously S100A16 and ZO-2 coexisted in the precipitated complexes obtained from SGC-7901 cells (Figure 6D), which was also observed in MGC-803 cells (Figure 6E). Consistently, immunofluorescence staining results revealed that S100A16 co-localized with ZO-2 in both two GC cell lines (Figure 6F). Using SGC-7901 cells, we determined the inverse correlation between ZO-2 and S100A16 in GC cells. S100A16 overexpression significantly reduced ZO-2 expression at the translational rather than transcriptional level (Figure 6G-H); by contrast, immunoblot results revealed the slight but obvious accumulation of ZO-2 in S100A16 knockdown SGC-7901 cells, although its mRNA levels did not significantly change (Figure 6I-J). Moreover, we observed that ZO-2 protein levels were obviously declined in GC cells compared with normal gastric mucosal cell lines (GES-1), which was inversely correlated with S100A16 expression (Figure 6K).

\section{S100A16-ZO-2 axis mediates migration, invasion and epithelial-mesenchymal transition (EMT) in GC cells}

It has been indicated that ZO-2 absence participates in the epithelial-mesenchymal transition (EMT), diffusely infiltrating growth, and ultimately frequent metastatic spread of GC cells [28], which is consistent with our observations that ZO-2 levels declined in GC patient's tumor tissues and correlated with poor survival rate (Figure S1A-D). To measure the role of ZO-2 in S100A16-induced GC cells metastasis, we performed several additional experiments. Results from wound healing assay indicated that the migratory speed was significantly enhanced in SGC-7901 cells and MGC cells that stably overexpressing lenti-S100A16 alone, but this enhancement could be reversed by ZO-2 replenishment (Figure 7A-B). Similarly, we applied tranwell assays and found that both migration and invasion ability was promoted by elevated S100A16 levels, which could also be reverted by ZO-2 overexpression (Figure 7C-D). Moreover, compared with lenti-scramble group, the concentrations of matrix metalloproteinases-2, 9 (MMP-2 and 9) in SGC-7901 stably overexpressing S100A16 culture supernatant obviously rose while tissue inhibitor of metalloproteinase-1 (TIMP-1) dramatically reduced, which was partially reversed by ZO2 supplement (Figure 7E), suggesting that S100A16-medicated ZO-2 inhibition contributes to GC invasion 
and metastasis. Notably, it has been widely recognized that the process of EMT facilitates tumor cells proliferation, motility and invasion during the tumor development, including gastric tumorigenesis and progression $[9,32,33]$ We next analyzed the protein levels of EMT markers including epithelial marker ECadherin, mesenchymal marker Vimentin as well as EMT promoting transcription factor Twist. As shown in Figure 7F-H, S100A16 overexpression alone downregulated E-Cadherin levels but upregulated Vimentin and Twist levels, which was barely seen in S100A16-ZO-2 overexpression group. Together, ZO-2 inhibition participates in the effects of S100A16 on invasion, migration and EMT phenotype of gastric cancer cells.

\section{The cellular content of ZO-2 diminishes after S100A16 overexpression due to ubiquitin-proteasome mediated degradation}

We then investigated the mechanism underlying S100A16-mediated ZO-2 inhibition. Given that S100A16 did not disturb ZO-2 mRNA levels in GC cells (Figure 6G-I), we speculated that excessive S100A16 may weaken the stability of ZO-2 proteins. As expected, S100A16 overexpression accelerated the decay of ZO2 proteins (Figure 8A). To figure out whether decreased ZO-2 was owning to proteasomal or lysosomal degradation, we applied cycloheximide (CHX) chase assays with MG132 (proteasomal degradation inhibitor) or chloroquine (lysosomal degradation inhibitor). As shown in Figure 8B-C, it was MG132 but not chloroquine that blocks the decline of ZO-2 protein levels induced by S100A16 overexpression. Consistently, S100A16 mediated the obvious increase in ZO-2 ubiquitination (Figure 8D). Interestingly, the lack of the Unique 2 (U2) region in ZO-2 diminished the pronounced ubiquitination caused by S100A16 (Figure 8D). Collectively, these results clearly demonstrate that S100A16 is responsible for ZO-2 ubiquitylation and degradation.

\section{Discussion}

Gastric cancer (GC) is one of the most common malignant tumors in the world, which poses a great threaten to human health. Globally, about $70 \%$ of gastric cancer cases occur in developing countries, especially in China. GC has been the fourth leading cause of cancer-related death, almost $7.39 \%$ of patients diagnosed with gastric cancer died from the disease every year [34]. The occurrence of gastric cancer is associated with multiple factors, including genetic factors, environmental factors. family history, diet, alcohol consumption, smoking, and Helicobacter pylori infection. The distant metastasis of tumors is one of the main reasons for the high recurrence and mortality rate of gastric cancer patients.

In this study, we explored the role of S100A16, a Ca ${ }^{2+}$-binding protein, in GC progression and metastasis. Analysis of databases and clinical samples reveals that S100A16 ${ }^{\text {high }}$ is positively correlated with poor prognosis and low overall survival rate of GC. Excessive S100A16 expression in GC cells leads to active proliferation and migration, whereas S100A16 inhibition effectively weakens the ability of growth and invasion in GC cells. When highly expressed, the interaction between S100A16 and ZO-2 (which acts as a core element in cell-to-cell tight junctions) was strengthened, and then contributed to the ubiquitination and degradation of ZO-2, weakening its biological function and eventually leading to elevated GC cells invasion and migration (Figure 8E). 
The S100 protein family, which belongs to the superfamily of $\mathrm{Ca}^{2+}$-binding proteins, has been reported to be involved in the progression of various cancers. S100A4 protein is one of the most extensively studied S100 family members, which is now considered as a strong likely biomarker for cancer diagnosis and metastasis prediction [35-37]. By contrast, expression of S100A8/S100A9 in sera has been reported to be associated with recurrence-free survival with bladder cancer [38]. According to previous studies, S100A16 is upregulated in bladder cancer, lung cancer, pancreatic cancer, colorectal cancer and ovary cancer [14, $15,17,39,40]$, but the role of S100A16 in GC has not been fully elucidated before. Our research demonstrated that S100A16 expression was significantly elevated in GC tissues, and GC cells with higher S100A16 expression levels tended to have more rapid speed of proliferation, migration and invasion. In vivo and in vitro analyses revealed that ectopic overexpression of S100A16 promotes tumor formation and migration, whereas S100A16 knockdown remarkably reduced tumor growth and migration. All of these observations reveal that S100A16 upregulation is both necessary and sufficient to trigger GC cells proliferation and invasion that ultimately leading to GC metastasis. However, the mechanism underlying S100A16 elevation in GC tumors is still unclear and remains to be investigated.

In the current studies, we applied mass spectrometry to figure out the molecular mechanisms underlying the pathological roles of S100A16 in GC. We investigated for the first time the interactive proteins of S100A16 in GC cells, and we found that tight junction-associated molecule Zona occludens-2 (ZO-2, also called TPJ2) bind directly with S100A16, and the interaction between those two proteins became stronger when S100A16 elevated. We observed that ZO-2 and S100A16 expression levels were inversely correlated in GC cells. In addition, ZO-2 replenishment significantly alleviated the accelerated GC cells invasion, migration and EMT caused by excessive S100A16.

ZO-2, a cytoplasmic protein of tight junctions, is a master regulator of gene expression, cell proliferation, cytoarchitecture, and cell size [25]. Studies have shown that ZO-2 expression is decreased in breast cancer, lung cancer, and other tumors [41-43]. It has been recognized that ZO-2 downregulation reduces the protective function of tight junctions between cells and related to tumor metastasis [24, 44], suggesting that ZO-2 may function as a tumor suppressor protein. Other studies have demonstrated that ZO-2 inhibition is involved in the diffusely infiltrating growth and frequent metastatic spread of GC cells $[28,29]$. Our research further clarifies the reason for declined ZO-2 in GC tumors, especially in tumor tissues with active proliferation and invasion, that is, S100A16 induces ZO-2 ubiquitination which is responsible for the abnormal ZO-2 degradation via ubiquitin-proteasomal system (UPS). In fact, ZO-2 could be degraded via both the ubiquitin-proteasomal or the lysosomal-mediated pathway, which depends on intracellular $\mathrm{Ca}^{2+}$ concentrations [45]. Interestingly, although S100A16 is a well-known Ca ${ }^{2+}$ binding protein, we observed that it bind with ZO-2 and mediated its degradation via UPS but not lysosomal pathway. UPS is one of the main mechanisms of cellular protein degradation. The degraded proteins are labeled through ubiquitination and then degraded by $26 \mathrm{~S}$ proteasomes. To combine ubiquitin with proteins, three distinct enzymes are need: E1 (ubiquitin-activating enzyme), E2 (ubiquitin-conjugating enzyme) and E3 (ubiquitin ligase); E3 ubiquitin ligases recognize certain protein substrates and catalyzes the transfer of activated ubiquitin to client proteins. The targeting specificity of proteins degraded by 
ubiquitin proteasomes is mediated by E3 ubiquitin ligases, which contains a large family of members [4649]. Obviously, no studies have shown that S100A16 could serve as one E3 ligase for targeting proteins to ubiquitinated. Therefore, we speculate that S100A16 may act as a scaffold or bridge protein between ZO2 and certain E3 ligase (s) that specifically recognize ZO-2. Back to our results, S100A16-induced ZO-2 ubiquitination was dramatically ameliorated when the unique 2 region (U2) of ZO-2 was deleted. Notably, it has been proved that the $\mathrm{U} 2$ region of ZO-2 is critical for the interaction with 14-3-3 proteins which protect ZO-2 from degradation via proteasomes [45]. Thus, it is highly possible that S100A16 recruits some E3 ligase (s) to bind with ZO-2 via its U2 region, and then forms a complex that leading to ZO-2 degradation.

\section{Conclusion}

In summary, our observations demonstrate that excessive S100A16-mediated Z0-2 ubiquitination and degradation play a crucial role in the progression of GC. This identification suggests that the therapeutic targeting of S100A16 could increase ZO-2 protein level to inhibit tumor invasion and metastasis for the treatment of GC.

\section{Abbreviations}

GC: Gastric cancer; S100A16: S100 Calcium Binding Protein A16; EMT: epithelial-to-mesenchymal transition; ZO-2: Zonula Occludens-2; TJs: Tight junctions.

\section{Declarations}

\section{Ethics approval and consent to participate}

The use of human tissue and experimental animals in this study was approved by the Ethics Committee and Institutional Animal Care and Use Committee of Nanjing Medical University. Informed consent was obtained from all patients.

\section{Consent for publication}

Not applicable.

\section{Availability of data and material}

The datasets generated during and/or analyzed during the current study are available from the corresponding authors upon reasonable request.

\section{Competing interests}

The authors declare no competing financial interests. 


\section{Funding}

Grants from the National Natural Science Foundation of China (81070656, 81170252 and 81570779) and Medical Research Project of Jiangsu Provincial Health and Family Planning Commission (H2019066) to D-M.S.; Grants from the National Natural Science Foundation of China (81670619 and 81870467) to X-B.L..

\section{Authors' contributions}

Conceptualization, D-M.S., and F.C.; Methodology, X-Y.Y., T-J.W., W-W.Z., M.L., and H-W. C.; Formal Analysis, D-M.S., F.C., and X-Y.Y.; Investigation, X-Y.Y., T-J.W., Y.H., W-J.G., and Y.L.; Resources, X-B.L., and D-M.S.; Writing-Original Draft, T-J.W.; Writing-Review \& Editing, D-M.S. and F.C.; Visualization, X-Y.Y.; Supervision, D-M.S. and F.C.; Funding Acquisition, D-M.S. and X-B.L.. All authors reviewed and commended on the manuscript.

\section{References}

1. Sitarz, R., et al., Gastric cancer: epidemiology, prevention, classification, and treatment. Cancer Manag Res, 2018. 10: p. 239-248.

2. Ferlay, J., et al., Cancer incidence and mortality worldwide: sources, methods and major patterns in GLOBOCAN 2012. Int J Cancer, 2015. 136(5): p. E359-86.

3. Ferlay, J., et al., Estimates of worldwide burden of cancer in 2008: GLOBOCAN 2008. Int J Cancer, 2010. 127(12): p. 2893-917.

4. Joshi, S.S. and B.D. Badgwell, Current treatment and recent progress in gastric cancer. CA Cancer J Clin, 2021. 71(3): p. 264-279.

5. Hamashima, C., Current issues and future perspectives of gastric cancer screening. World $\mathrm{J}$ Gastroenterol, 2014. 20(38): p. 13767-74.

6. Smyth, E.C., et al., Gastric cancer. Lancet, 2020. 396(10251): p. 635-648.

7. Feng, W., et al., Non-coding RNAs in regulating gastric cancer metastasis. Clin Chim Acta, 2019. 496: p. 125-133.

8. Zhao, J., et al., Long non-coding RNA Linc00152 is involved in cell cycle arrest, apoptosis, epithelial to mesenchymal transition, cell migration and invasion in gastric cancer. Cell Cycle, 2015. 14(19): p. 311223.

9. Peng, Z., et al., Role of epithelial-mesenchymal transition in gastric cancer initiation and progression. World J Gastroenterol, 2014. 20(18): p. 5403-10. 
10. Bresnick, A.R., D.J. Weber, and D.B. Zimmer, S100 proteins in cancer. Nat Rev Cancer, 2015. 15(2): p. 96-109.

11. Yap, K.L., et al., Diversity of conformational states and changes within the EF-hand protein superfamily. Proteins, 1999. 37(3): p. 499-507.

12. Zimmer, D.B. and D.J. Weber, The Calcium-Dependent Interaction of S100B with Its Protein Targets. Cardiovasc Psychiatry Neurol, 2010. 2010.

13. Ji, Y.F., et al., $\mathrm{S} 100$ family signaling network and related proteins in pancreatic cancer (Review). Int $\mathrm{J}$ Mol Med, 2014. 33(4): p. 769-76.

14. Fang, D., et al., S100A16 promotes metastasis and progression of pancreatic cancer through FGF19mediated AKT and ERK1/2 pathways. Cell Biol Toxicol, 2021.

15. Zhuang, H., et al., Prognostic values and immune suppression of the S100A family in pancreatic cancer. J Cell Mol Med, 2021. 25(6): p. 3006-3018.

16. Zhang, J., et al., S100A16 suppresses the growth and survival of leukaemia cells and correlates with relapse and relapse free survival in adults with Philadelphia chromosome-negative B-cell acute lymphoblastic leukaemia. Br J Haematol, 2019. 185(5): p. 836-851.

17. Zhou, W., et al., Up-regulation of S100A16 expression promotes epithelial-mesenchymal transition via Notch1 pathway in breast cancer. J Biomed Sci, 2014. 21: p. 97.

18. Lv, H., et al., MicroRNA-6884-5p Regulates the Proliferation, Invasion, and EMT of Gastric Cancer Cells by Directly Targeting S100A16. Oncol Res, 2020. 28(3): p. 225-236.

19. Jiang, Y., et al., ADAMTS19 Suppresses Cell Migration and Invasion by Targeting S100A16 via the NFkappaB Pathway in Human Gastric Cancer. Biomolecules, 2021. 11(4).

20. Martin, T.A. and W.G. Jiang, Loss of tight junction barrier function and its role in cancer metastasis. Biochim Biophys Acta, 2009. 1788(4): p. 872-91.

21. Bhat, A.A., et al., Tight Junction Proteins and Signaling Pathways in Cancer and Inflammation: A Functional Crosstalk. Front Physiol, 2018. 9: p. 1942.

22. Lauko, A., et al., Junctional Adhesion Molecules in Cancer: A Paradigm for the Diverse Functions of Cell-Cell Interactions in Tumor Progression. Cancer Res, 2020. 80(22): p. 4878-4885.

23. Umeda, K., et al., ZO-1 and ZO-2 independently determine where claudins are polymerized in tightjunction strand formation. Cell, 2006. 126(4): p. 741-54.

24. Akizuki, R., et al., ZO-2 Suppresses Cell Migration Mediated by a Reduction in Matrix Metalloproteinase 2 in Claudin-18-Expressing Lung Adenocarcinoma A549 Cells. Biol Pharm Bull, 2019. 
42(2): p. 247-254.

25. Gonzalez-Mariscal, L., et al., ZO-2 Is a Master Regulator of Gene Expression, Cell Proliferation, Cytoarchitecture, and Cell Size. Int J Mol Sci, 2019. 20(17).

26. Cong, Y., et al., Tim-3 promotes cell aggressiveness and paclitaxel resistance through NFkappaB/STAT3 signalling pathway in breast cancer cells. Chin J Cancer Res, 2020. 32(5): p. 564-579.

27. Yang, C.C., et al., Plant galactolipid dLGG suppresses lung metastasis of melanoma through deregulating TNF-alpha-mediated pulmonary vascular permeability and circulating oxylipin dynamics in mice. Int J Cancer, 2018. 143(12): p. 3248-3261.

28. Shinto, O., et al., Inhibitory effect of a TGFbeta receptor type-l inhibitor, Ki26894, on invasiveness of scirrhous gastric cancer cells. Br J Cancer, 2010. 102(5): p. 844-51.

29. Kato, Y., et al., Establishment and characterization of a new hypoxia-resistant cancer cell line, OCUM12/Hypo, derived from a scirrhous gastric carcinoma. Br J Cancer, 2010. 102(5): p. 898-907.

30. Fan, Y., et al., CircNR3C2 promotes HRD1-mediated tumor-suppressive effect via sponging miR-513a$3 p$ in triple-negative breast cancer. Mol Cancer, 2021. 20(1): p. 25.

31. Wu, T., et al., HRD1, an Important Player in Pancreatic beta-Cell Failure and Therapeutic Target for Type 2 Diabetic Mice. Diabetes, 2020. 69(5): p. 940-953.

32. Christiansen, J.J. and A.K. Rajasekaran, Reassessing epithelial to mesenchymal transition as a prerequisite for carcinoma invasion and metastasis. Cancer Res, 2006. 66(17): p. 8319-26.

33. Natalwala, A., R. Spychal, and C. Tselepis, Epithelial-mesenchymal transition mediated tumourigenesis in the gastrointestinal tract. World J Gastroenterol, 2008. 14(24): p. 3792-7.

34. Wu, H., et al., The role and application of small extracellular vesicles in gastric cancer. Mol Cancer, 2021. 20(1): p. 71.

35. Kim, Y.J., et al., Metastasis-associated protein S100A4 and p53 predict relapse in curatively resected stage III and IV (M0) gastric cancer. Cancer Invest, 2008. 26(2): p. 152-8.

36. Wang, Y.Y., et al., High-level expression of S100A4 correlates with lymph node metastasis and poor prognosis in patients with gastric cancer. Ann Surg Oncol, 2010. 17(1): p. 89-97.

37. Tang, Z., et al., Contribution of upregulated dipeptidyl peptidase 9 (DPP9) in promoting tumoregenicity, metastasis and the prediction of poor prognosis in non-small cell lung cancer (NSCLC). Int J Cancer, 2017. 140(7): p. 1620-1632.

38. Minami, S., et al., Proteomic study of sera from patients with bladder cancer: usefulness of S100A8 and S100A9 proteins. Cancer Genomics Proteomics, 2010. 7(4): p. 181-9. 
39. Bai, Y., et al., Prognostic values of $\mathrm{S} 100$ family members in ovarian cancer patients. BMC Cancer, 2018. 18(1): p. 1256.

40. Sun, X., et al., S100A16 is a prognostic marker for colorectal cancer. J Surg Oncol, 2018. 117(2): p. 275-283.

41. Martin, T.A., et al., Loss of tight junction plaque molecules in breast cancer tissues is associated with a poor prognosis in patients with breast cancer. Eur J Cancer, 2004. 40(18): p. 2717-25.

42. Paschoud, S., et al., Claudin-1 and claudin-5 expression patterns differentiate lung squamous cell carcinomas from adenocarcinomas. Mod Pathol, 2007. 20(9): p. 947-54.

43. Gao, F., et al., Endothelial Akt1 loss promotes prostate cancer metastasis via beta-catenin-regulated tight-junction protein turnover. Br J Cancer, 2018. 118(11): p. 1464-1475.

44. Luczka, E., et al., Regulation of membrane-type 1 matrix metalloproteinase expression by zonula occludens-2 in human lung cancer cells. Clin Exp Metastasis, 2013. 30(7): p. 833-43.

45. Amaya, E., et al., Activation of the $\mathrm{Ca}(2+)$ sensing receptor and the PKC/WNK4 downstream signaling cascade induces incorporation of ZO-2 to tight junctions and its separation from 14-3-3. Mol Biol Cell, 2019. 30(18): p. 2377-2398.

46. Bernassola, F., G. Chillemi, and G. Melino, HECT-Type E3 Ubiquitin Ligases in Cancer. Trends Biochem Sci, 2019. 44(12): p. 1057-1075.

47. Senft, D., J. Qi, and Z.A. Ronai, Ubiquitin ligases in oncogenic transformation and cancer therapy. Nat Rev Cancer, 2018. 18(2): p. 69-88.

48. Buetow, L. and D.T. Huang, Structural insights into the catalysis and regulation of E3 ubiquitin ligases. Nat Rev Mol Cell Biol, 2016. 17(10): p. 626-42.

49. Fujita, Y., et al., Ubiquitin Ligases in Cancer Immunotherapy - Balancing Antitumor and Autoimmunity. Trends Mol Med, 2019. 25(5): p. 428-443.

\section{Tables}

Table 1. Clinicopathological characteristics GC patients in purchased microarray 


\begin{tabular}{|c|c|}
\hline Characteristics & Cases \\
\hline \multicolumn{2}{|l|}{ Clinical information } \\
\hline Median age years [(range)] & $65 \rrbracket 32-81 \rrbracket$ \\
\hline Gender囚Male/Female】 & $\llbracket 64 / 36 \rrbracket$ \\
\hline \multicolumn{2}{|l|}{ Characteristics of tumour } \\
\hline Median volume of tumor [cm(range)] & $5 \bigotimes 1.2-20 \rrbracket$ \\
\hline Pathological grade $\mathbb{X} / \mathbb{\Delta} / \mathbb{Q}$ & $13 / 76 / 11$ \\
\hline Vascular invasion $₫$ Yes/No $\rrbracket$ & $14 / 86$ \\
\hline 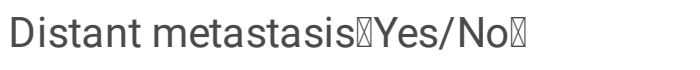 & $8 / 92$ \\
\hline \multicolumn{2}{|l|}{ Pathological features } \\
\hline Differentiation $₫$ high/media/low $\rrbracket$ & $15 / 74 / 11$ \\
\hline Median survival time [month(range)] & $24 \rrbracket 1-107 \rrbracket$ \\
\hline Survival cases & 28 \\
\hline Death cases & 69 \\
\hline Loss of follow-up & 3 \\
\hline
\end{tabular}

Table 2. Distribution of each factor for S100A $16 A^{\text {high }}$ and S100A $16^{\text {low }}$ groups 


\begin{tabular}{|c|c|c|c|c|}
\hline & low expression & high expression & \multirow[t]{2}{*}{ Statistics } & \multirow[t]{2}{*}{ P-value } \\
\hline & $(\mathrm{N}=72)$ & $(\mathrm{N}=25)$ & & \\
\hline \multicolumn{5}{|l|}{ Gender } \\
\hline Male & 49 & 13 & \multirow[t]{2}{*}{$x 2=2.31$} & \multirow[t]{2}{*}{0.126} \\
\hline Female & 23 & 12 & & \\
\hline \multicolumn{5}{|c|}{ Pathological grade } \\
\hline ૫ & 7 & 4 & \multirow[t]{3}{*}{$\omega=1022.5$} & \multirow[t]{3}{*}{0.29} \\
\hline ૫ & 56 & 20 & & \\
\hline ૫ & 9 & 1 & & \\
\hline Tumor size(cm) & $5.83(3.64)^{\mathrm{a}}$ & $6.55(3.32)$ & $t=-0.91$ & 0.37 \\
\hline Ki67 & $0.16 \rrbracket 0.21 \rrbracket$ & $0.23 \bowtie 0.29 \rrbracket$ & $\omega=640.5$ & 0.032 \\
\hline
\end{tabular}

Table 3. Multivariate Cox regression fitting results

\begin{tabular}{|lllll|}
\hline Variable & Estimated effect & HR & $95 \% \mathrm{Cl}$ & P value \\
\hline Expression & 0.986 & 2.681 & $1.545 \sim 4.643$ & 0.00043 \\
\hline Gender & -0.107 & 0.899 & $0.535 \sim 1.510$ & 0.686 \\
\hline Pathological grade & 0.649 & 1.914 & $1.095 \sim 3.345$ & 0.0228 \\
\hline Tumour size & 0.079 & 1.082 & $1.011 \sim 1.159$ & 0.0239 \\
\hline Ki67 & -0.389 & 0.678 & $0.252 \sim 1.824$ & 0.4415 \\
\hline
\end{tabular}

Table 4. List of the interactive proteins of S100A16 in SGC-7901 cells analyzed by mass spectrometry. 


\begin{tabular}{|c|c|c|c|c|}
\hline Gene & Protein & ${ }^{a} \mathrm{NC}$ & bS100A16 & $\begin{array}{l}\text { b/a } \\
\text { ratio }\end{array}$ \\
\hline PRSS1 & Trypsin-1 (Fragment) & + & - & \\
\hline MTFR1 & Mitochondrial fission regulator 1 (Fragment) & + & - & \\
\hline ZNF280A & Suppressor of hairy wing homolog 1 (Drosophila) & + & - & \\
\hline HIST1H4A & Histone H4 & + & - & \\
\hline KAT5 & Histone acetyltransferase KAT5 (Fragment) & + & - & \\
\hline CARD18 & Caspase recruitment domain-containing protein 18 & + & - & \\
\hline ERF & $\begin{array}{l}\text { ETS domain-containing transcription factor } \\
\text { ERF(Fragment) }\end{array}$ & + & - & \\
\hline EEF1A1P5 & Putative elongation factor 1 -alpha-like 3 & + & - & \\
\hline PELO & Protein pelota homolog & + & - & \\
\hline SERPINA1 & Alpha-1-antitrypsin (Fragment) & + & - & \\
\hline PMEPA1 & Isoform 3 of Protein TMEPAI & + & - & \\
\hline DNAH10 & Dynein heavy chain 10 , axonemal & + & - & \\
\hline TNRC6A & $\begin{array}{l}\text { Trinucleotide repeat-containing gene } 6 \mathrm{~A} \text { protein } \\
\text { (Fragment) }\end{array}$ & + & - & \\
\hline MAGEB3 & Melanoma-associated antigen B3 & + & - & \\
\hline ZNF184 & Zinc finger protein 184 & + & - & \\
\hline SLC8A3 & Isoform 8 of Sodium/calcium exchanger 3 & + & - & \\
\hline HIST1H2BN & Histone $\mathrm{H} 2 \mathrm{~B}$ & + & - & \\
\hline DSG1 & Desmoglein-1 & + & - & \\
\hline POLE2 & Isoform 2 of DNA polymerase epsilon subunit 2 & + & - & \\
\hline CARMIL1 & F-actin-uncapping protein LRRC16A (Fragment) & + & - & \\
\hline FLNA & Filamin-A & + & - & \\
\hline AlM1 & Absent in melanoma 1 protein & + & - & \\
\hline CNTRL & Isoform 3 of Centriolin & + & + & 0 \\
\hline TJP2 & Tight junction protein ZO-2 & + & + & 0.01 \\
\hline DNAH3 & Dynein heavy chain 3 , axonemal & + & + & 0.04 \\
\hline ROS1 & Tyrosine-protein kinase receptor & + & + & 0.15 \\
\hline JUP & Junction plakoglobin & + & + & 0.2 \\
\hline
\end{tabular}




\begin{tabular}{|c|c|c|c|c|}
\hline S100A8 & Protein S100-A8 & + & + & 0.44 \\
\hline HSP90AB1 & Heat shock protein HSP 90-beta & + & + & 0.51 \\
\hline PRCP & Lysosomal Pro-X carboxypeptidase (Fragment) & - & + & \\
\hline SERPINF2 & Alpha-2-antiplasmin (Fragment) & - & + & \\
\hline ACTBL2 & Beta-actin-like protein 2 & - & + & \\
\hline $\mathrm{A} 2 \mathrm{M}$ & Alpha-2-macroglobulin & - & + & \\
\hline MLEC & Malectin (Fragment) & - & + & \\
\hline MYH9 & Myosin-9 & - & + & \\
\hline RGS13 & Regulator of G-protein signaling 13 & - & + & \\
\hline KCNIP4 & Isoform 3 of $\mathrm{Kv}$ channel-interacting protein 4 & - & + & \\
\hline PSMB1 & Proteasome subunit beta type- 1 & - & + & \\
\hline DRP2 & Isoform 2 of Dystrophin-related protein 2 & - & + & \\
\hline EXD3 & Exonuclease mut-7 homolog, isoform 5 & - & + & \\
\hline KLHL38 & Kelch-like protein 38 & - & + & \\
\hline ATP5B & ATP synthase subunit beta (Fragment) & - & + & \\
\hline CNR1 & Isoform 2 of Cannabinoid receptor 1 & - & + & \\
\hline KIAA2012 & Uncharacterized protein KIAA2012 & - & + & \\
\hline HSPA1A & Isoform 2 of Heat shock $70 \mathrm{kDa}$ protein $1 \mathrm{~A}$ & - & + & \\
\hline SBSN & Suprabasin & - & + & \\
\hline MYOZ2 & Myozenin-2 & - & + & \\
\hline СCT5 & T-complex protein 1 subunit epsilon & - & + & \\
\hline NSD1 & Isoform 2 of Histone-lysine N-methyltransferase, $\mathrm{H} 3$ & - & + & \\
\hline DDX21 & Isoform 2 of Nucleolar RNA helicase 2 & - & + & \\
\hline S100A16 & Protein S100-A16 & + & + & 22.05 \\
\hline MSI2 & RNA-binding protein Musashi homolog 2 (Fragment) & + & + & 13.07 \\
\hline APOE & Apolipoprotein E & + & + & 11.37 \\
\hline CAPNS1 & Calpain small subunit 1 (Fragment) & + & + & 5.92 \\
\hline HSPA9 & Stress-70 protein, mitochondrial & + & + & 4.83 \\
\hline HNRNPU & $\begin{array}{l}\text { Isoform Short of Heterogeneous nuclear } \\
\text { ribonucleoprotein U }\end{array}$ & + & + & 4.33 \\
\hline
\end{tabular}




\begin{tabular}{|llccc|}
\hline DSP & Desmoplakin & + & + & 2.69 \\
\hline C3 & Complement C3 & + & + & 2.26 \\
\hline APOA1 & Apolipoprotein A-I & + & + & 2.19 \\
\hline SERPINC1 & Antithrombin-III & + & + & 2.15 \\
\hline PZP & Pregnancy zone protein & + & + & 2.08 \\
\hline ALB & Serum albumin & + & + & 2.04 \\
\hline TUBB & Tubulin beta chain & + & + & 1.95 \\
\hline C4A & Complement C4-A & + & + & 1.89 \\
\hline HRNR & Hornerin & + & + \\
\hline KCTD3 & $\begin{array}{l}\text { Isoform 2 of BTB/POZ domain-containing protein } \\
\text { KCTD3 }\end{array}$ & + & + \\
\hline ATP5A1 & $\begin{array}{l}\text { Isoform 2 of ATP synthase subunit alpha, } \\
\text { mitochondrial }\end{array}$ & + & 1.71 \\
\hline a SGC-7901 cells infected with negative control lentivirus. & + & \\
\hline b SGC-7901 cells infected with lentivirus overexpressing S100A16. & + & \\
\hline
\end{tabular}

\section{Figures}




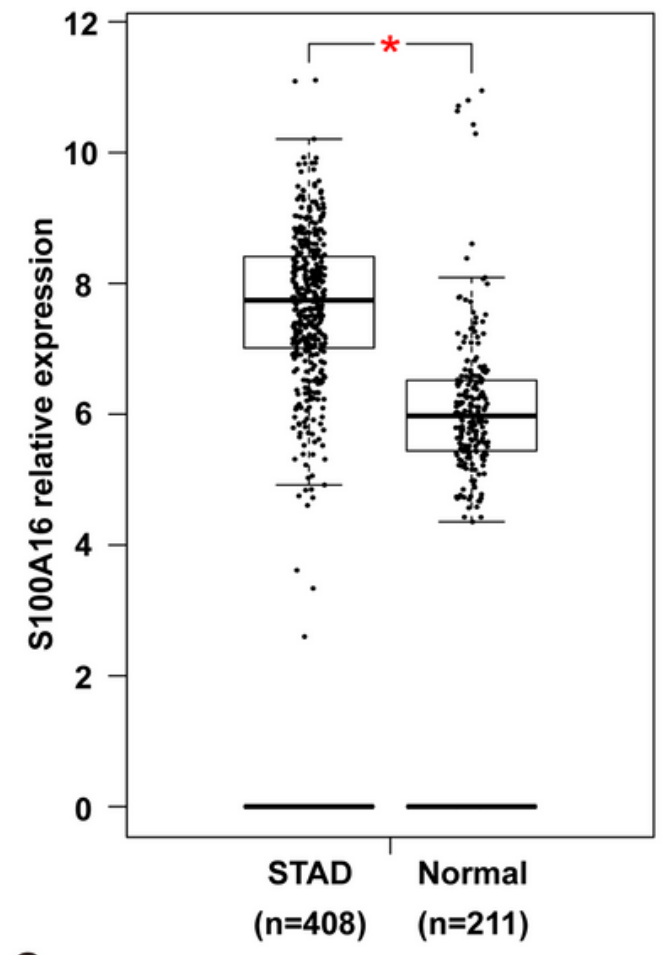

C

S100A16 expression in STAD based on H.pylori infection status

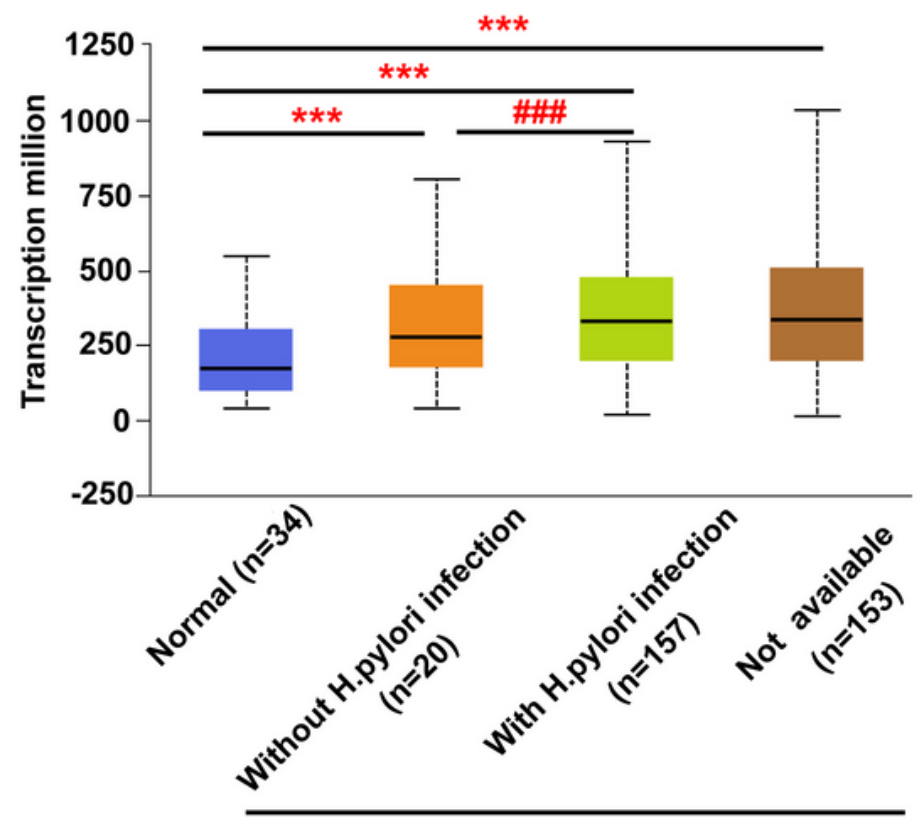

STAD

\section{S100A16 expression in STAD based on tissue types}

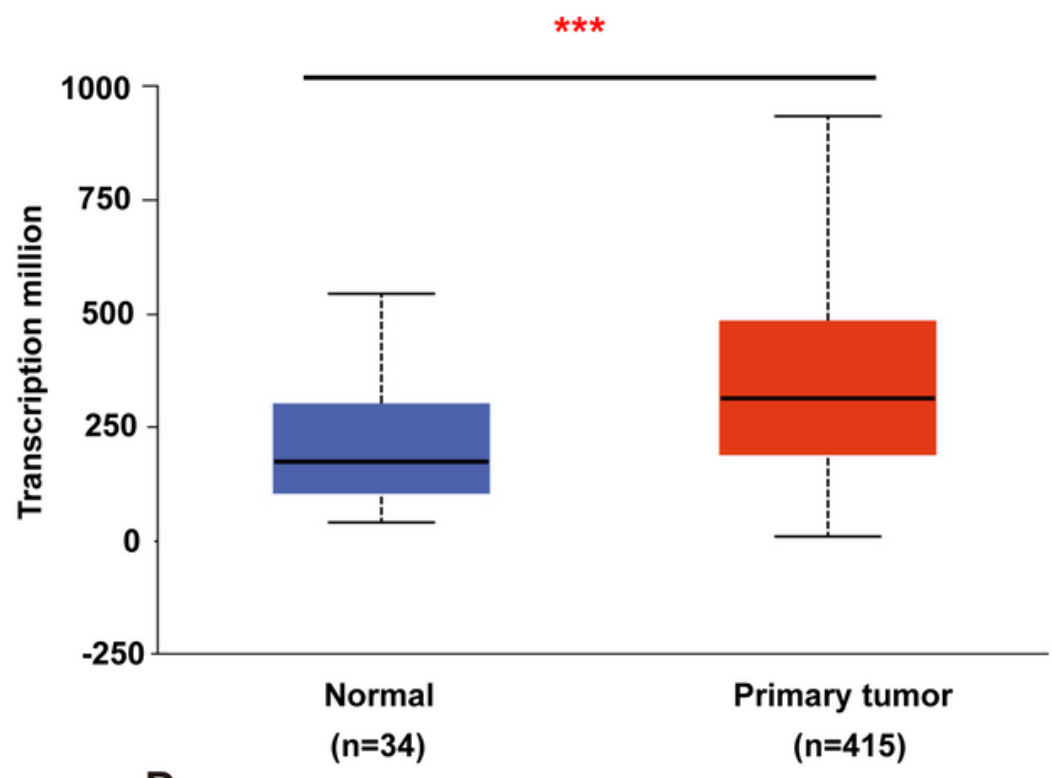

D

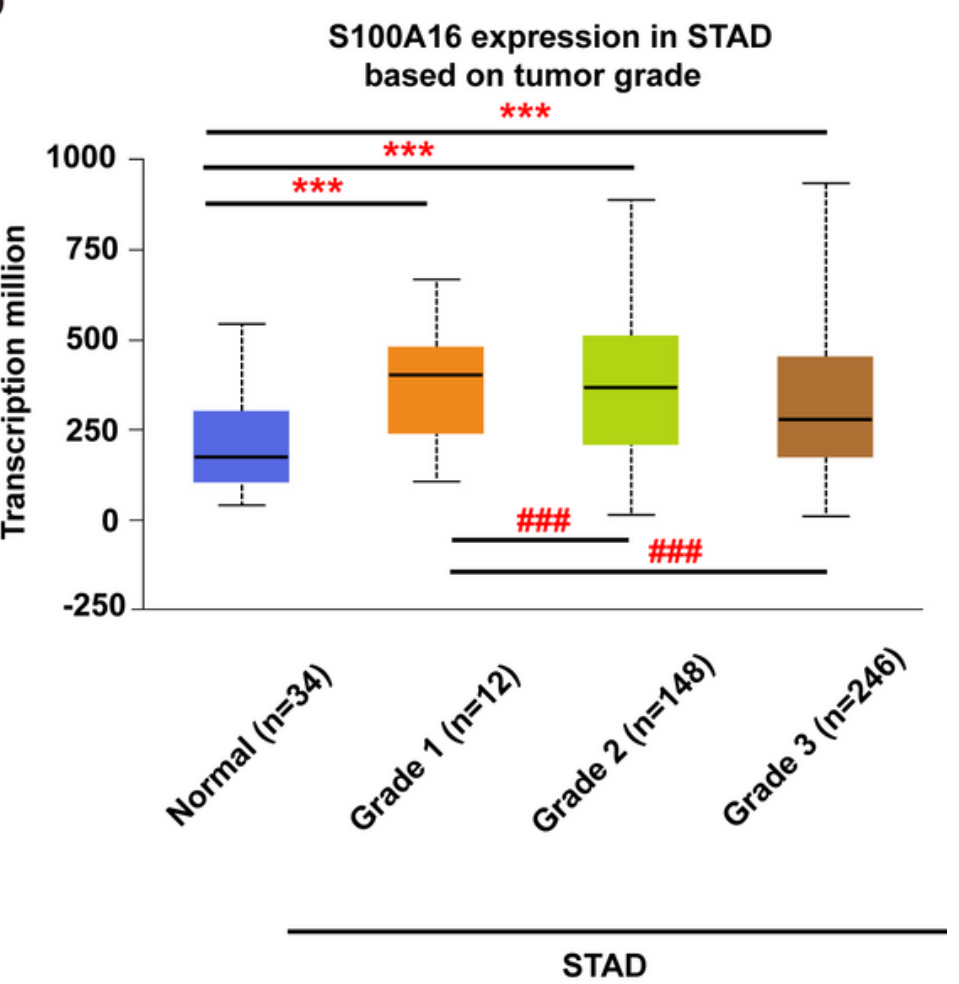

Figure 1

Bioinformatic analysis S100A16 expression in GC. (A-B) S100A16 expression in human GC tissues according to GEPIA and UALCAN database. (C) Boxplot showing expression of S100A16 associated with $\mathrm{H}$. pylori infection status. (D) Boxplot showing relative expression of S100A16 in normal individuals or GC

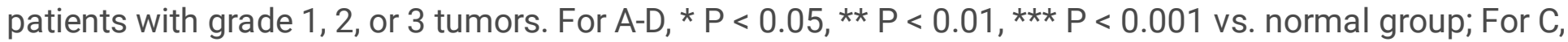
\#\#\# $\mathrm{P}<0.001$ vs. STAD without H. pylori infection; For D, \#\#\# $\mathrm{P}<0.001$ vs. Grade 1 STAD. 
A

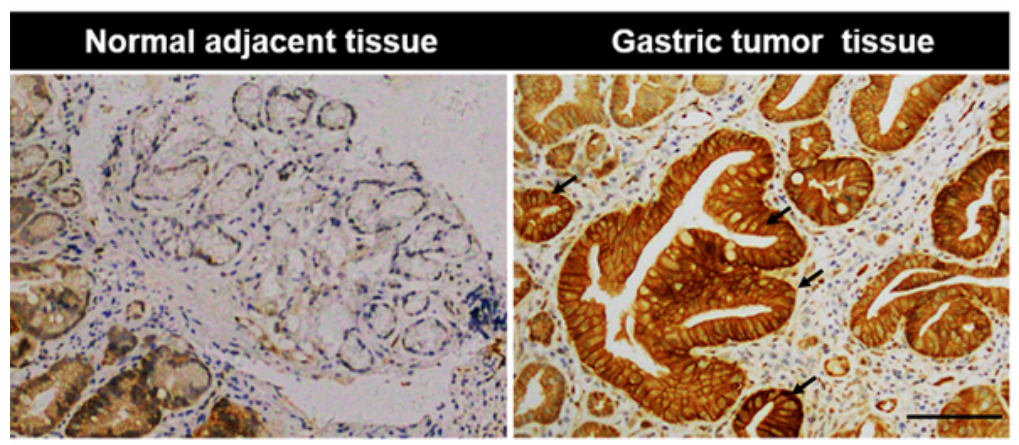

C

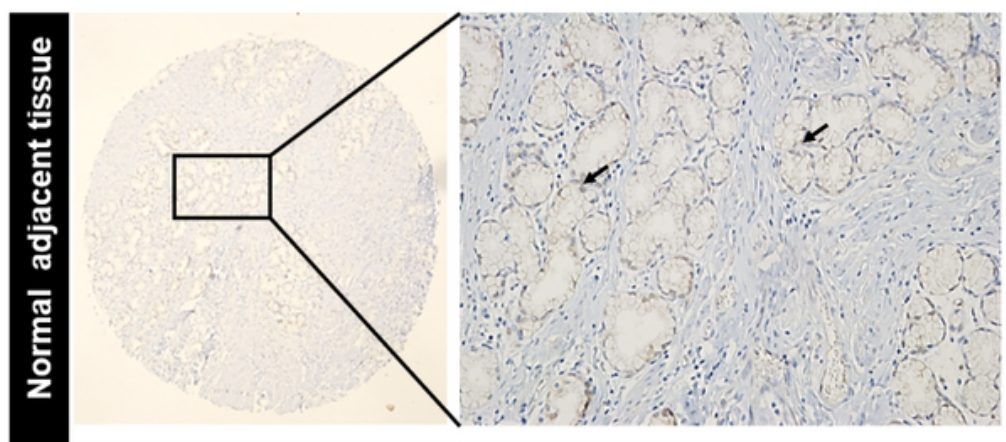

흘

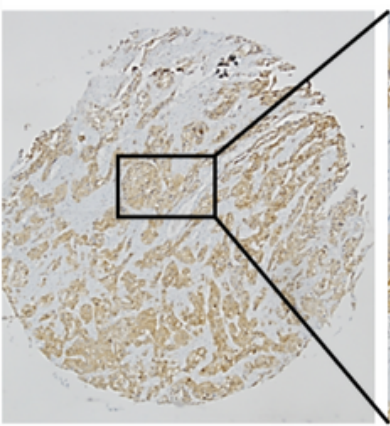

E

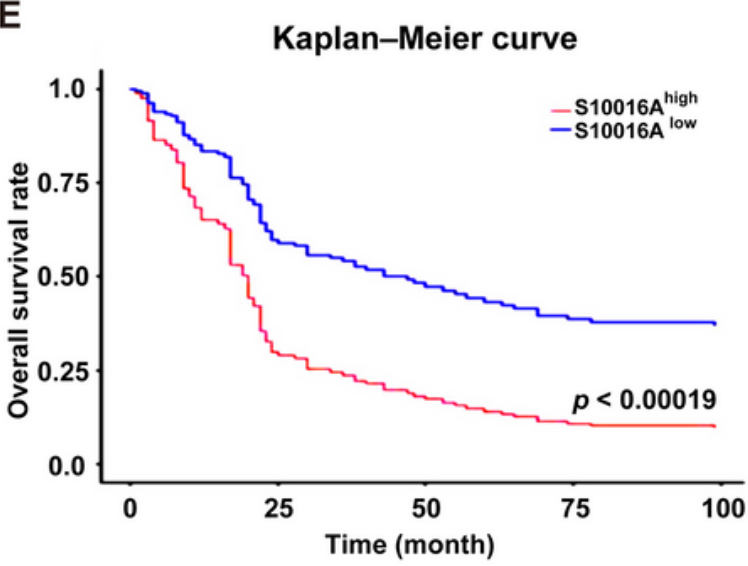

B

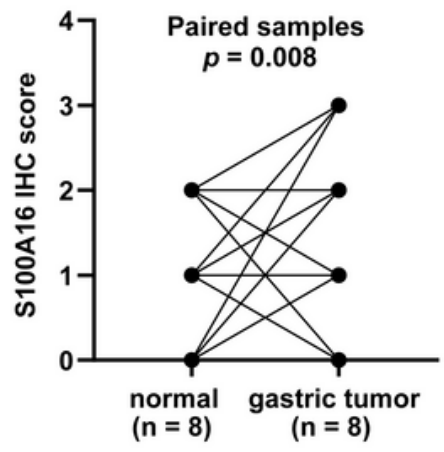

D
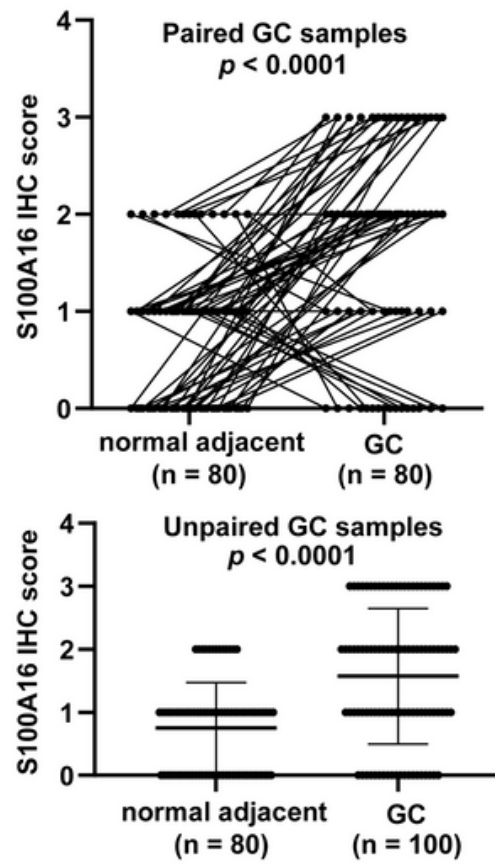

F

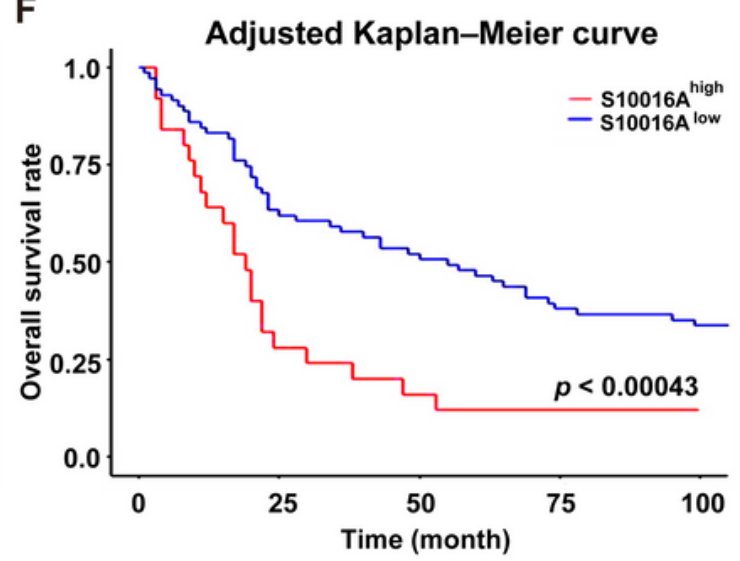

Figure 2

S100A16 is upregulated in GC tissues and correlated with poor prognosis in GC patients. (A) IHC staining of S100A16 in gastric tumors compared with normal adjacent tissues obtained from the Department of Pathology in Sir Run-Run Hospital of Nanjing Medical University. Scare bar = $200 \mu \mathrm{m}$. (B) Quantification of immunohistochemical staining in paired gastric samples $(n=8)$. (C) IHC staining of S100A16 in GC tissues compared with normal adjacent tissues, both of which obtained from purchased tissue 
microarray. Scare bar $=200 \mu \mathrm{m}$. (D) Paired line plots showing S100A16 IHC score in matched gastric cancer tissues ( $n=80$, upper). Unpaired line plots showing S100A16 IHC score in gastric cancer tissues (normal adjacent tissues ( $n=80)$ GC tissues $(n=100)$, lower). (E) Kaplan-Meier survival analysis of S100A16high and S100A16low GC patients mentioned in (C). (F) Adjusted Kaplan-Meier curves of overall survival rate in (E).

A

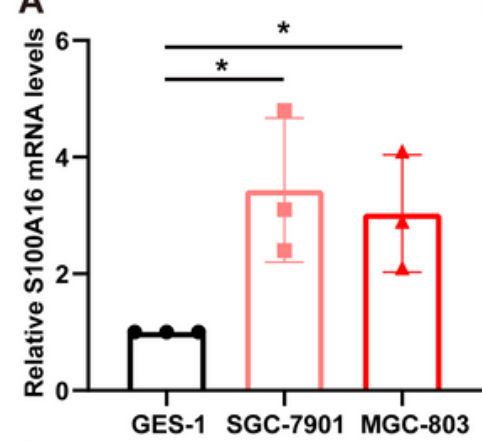

D

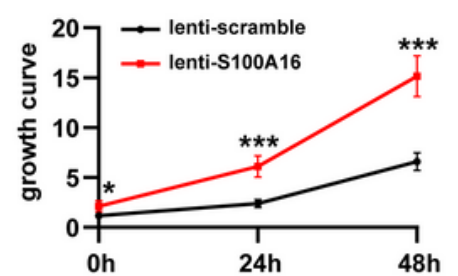

G

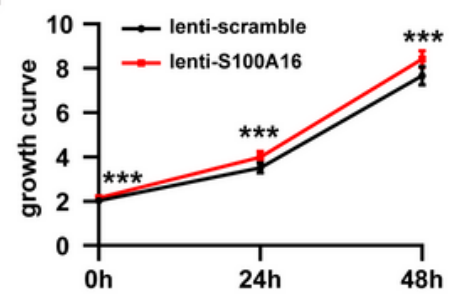

J

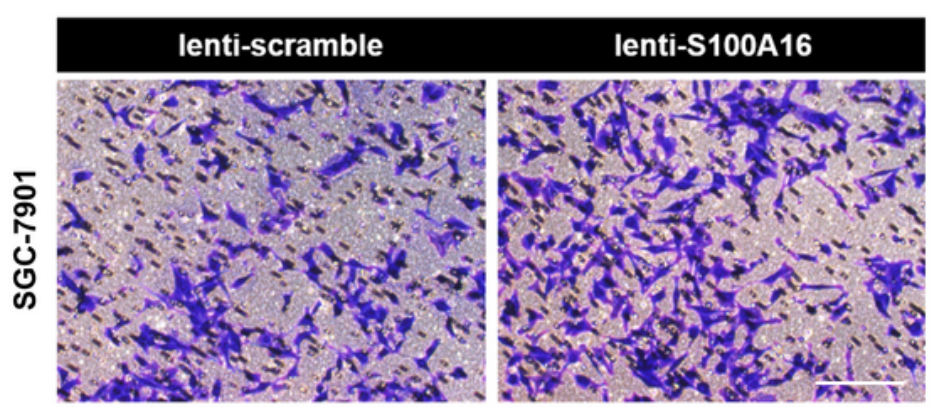

$\mathbf{L}$

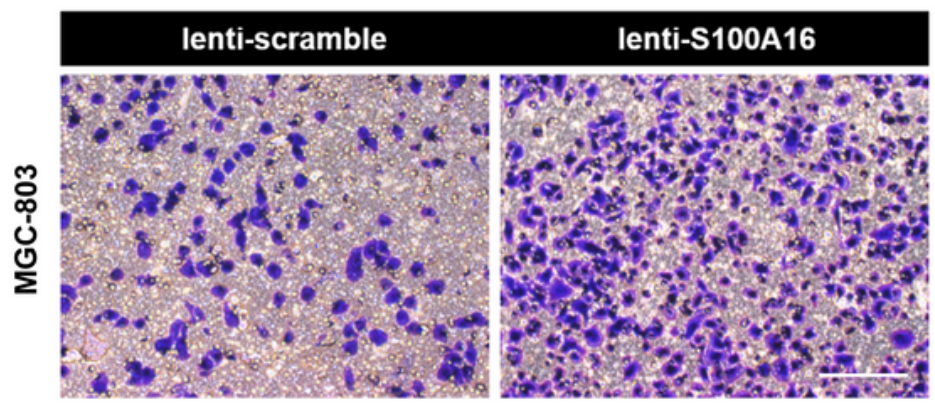

E

SGC-7901

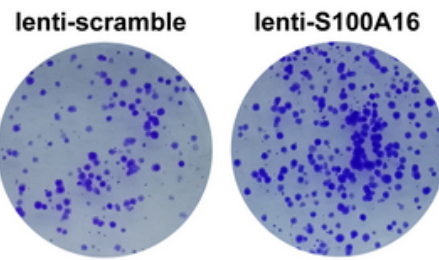

$\mathrm{H}$
B C
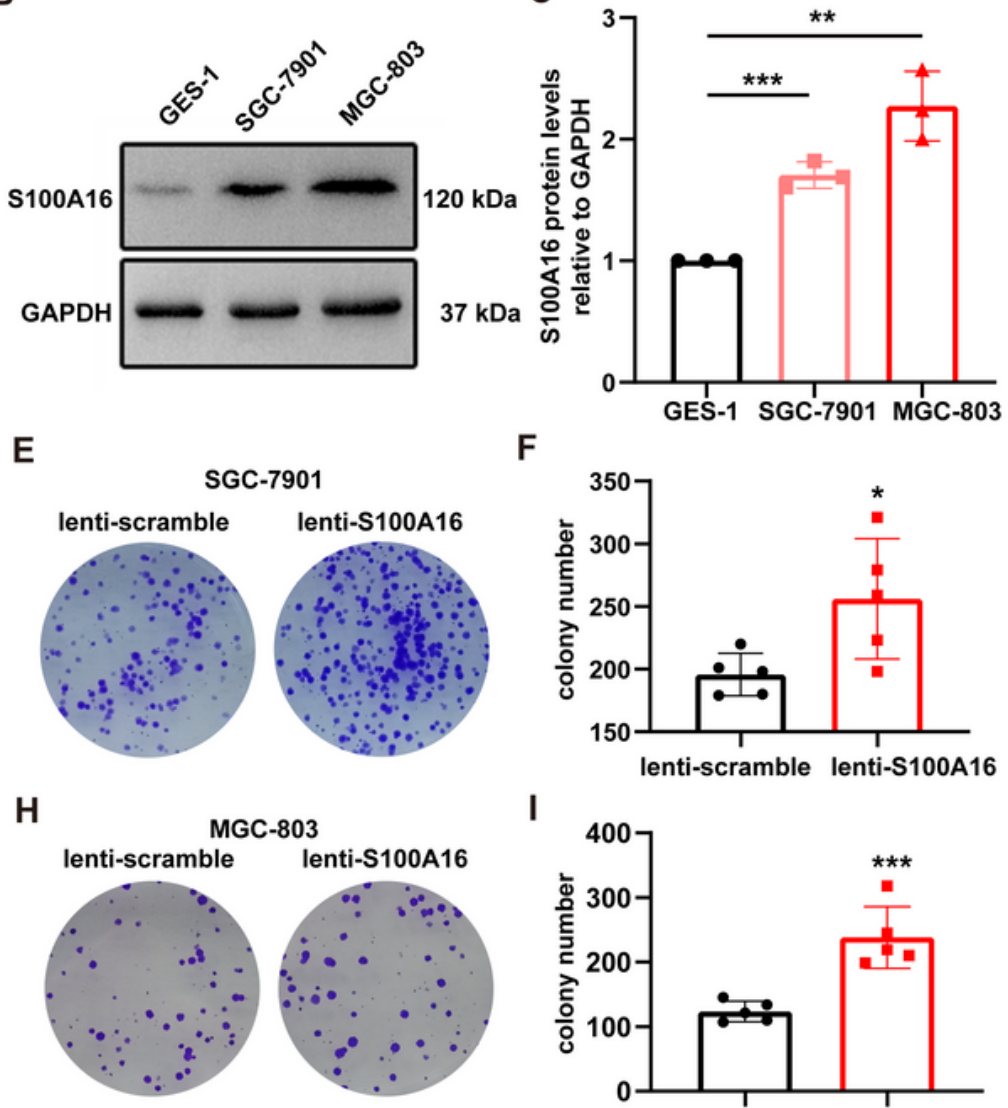

$\mathbf{F}$
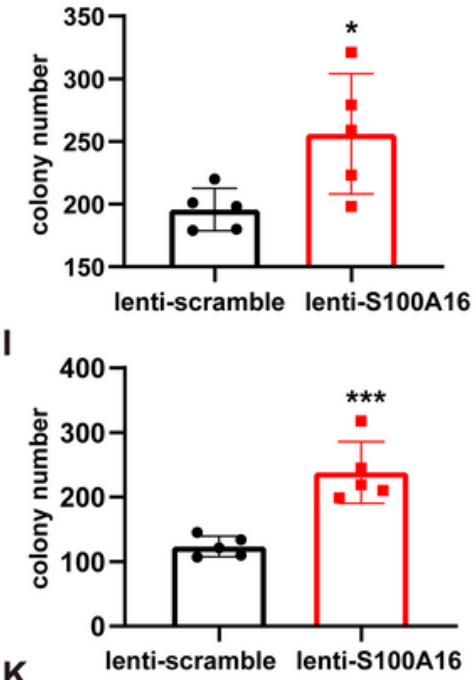

K

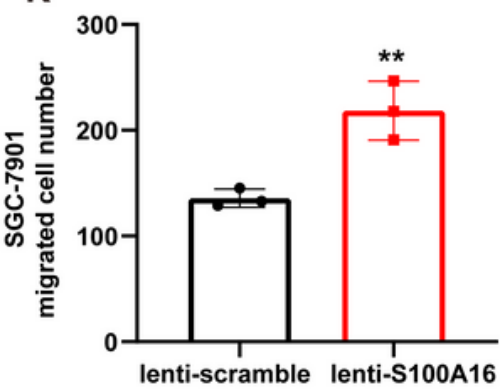

M

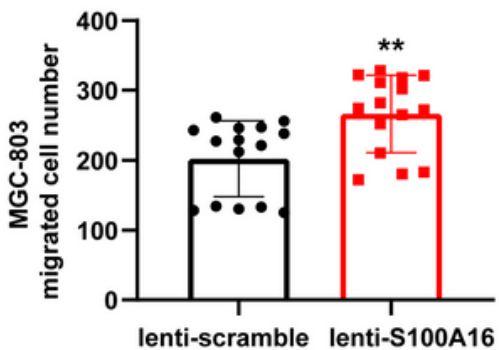

Figure 3 
Overexpression of S100A16 accelerates GC cells growth and migration in vitro. (A-B) The mRNA and protein levels of S100A16 in GES-1, SGC-7901, and MGC-803 cells. GAPDH was used as internal standard. (C) Grey density of (B). (D) Cell growth was measured by SRB staining in SGC-7901 cells stably overexpressing S100A16. (E) Colonies formed by SGC-7901 cells that stably overexpressing S100A16. (F) Colonies number in (E) were quantified. (G) Cell growth was measured by SRB staining in MGC-803 cells stably overexpressing S100A16. (H) Colonies formed by MGC-803 cells that stably overexpressing S100A16. (I) Colonies number in (H) were quantified. (J-K) The migration ability of SGC-7901 cells stably overexpressing S100A16 was measured by transwell assay. Migrated cell numbers were counted in (K). Scare bar $=100 \mu \mathrm{m}$. (L-M) The migration ability of MGC-803 cells stably overexpressing S100A16 was measured by transwell assay. Migrated cell numbers were counted in $(\mathrm{M})$. Scare bar $=100 \mu \mathrm{m}$. Data are presented as mean $\pm S D . n=3-15$ for each group. For $A$ and $C$, $* P<0.05$, $* \star P<0.01, \star \star \star ~ P<0.001$ vs. GES-1 cells. For $D, F, G, I, K$ and $M, * P<0.05$, ${ }^{\star *} P<0.01$, ${ }^{\star * *} P<0.001$ vs. lenti-scramble group. 
A

SGC-7901 cells

(lenti-S100A16 or -control group)

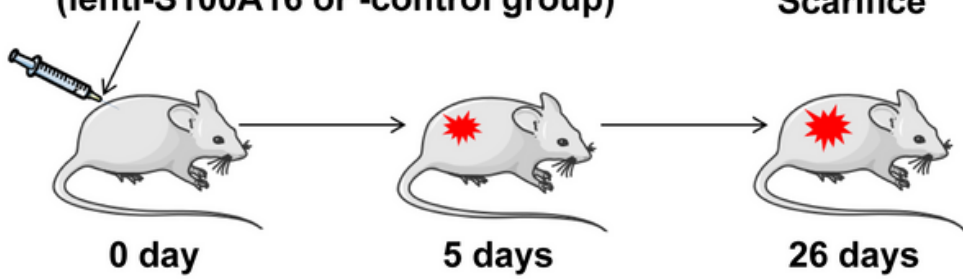

C

\section{lenti-control}

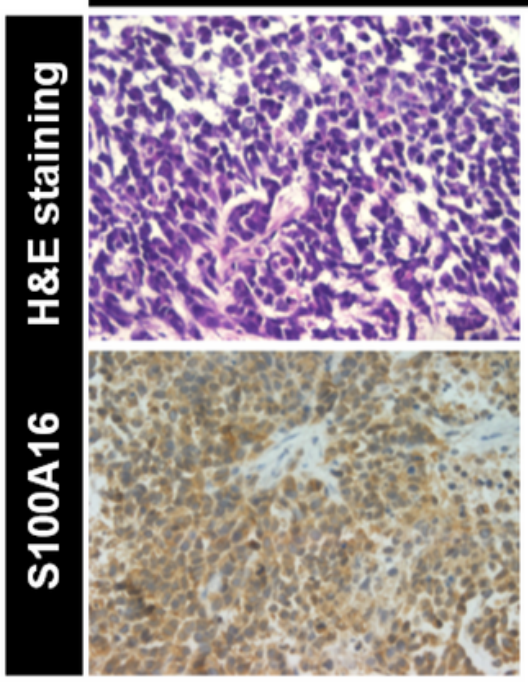

E

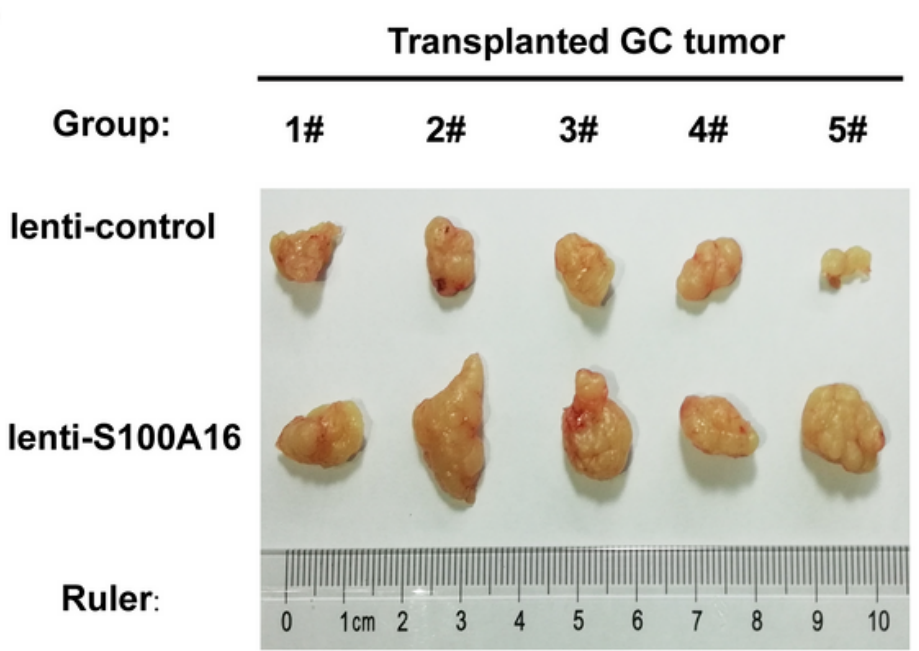

B
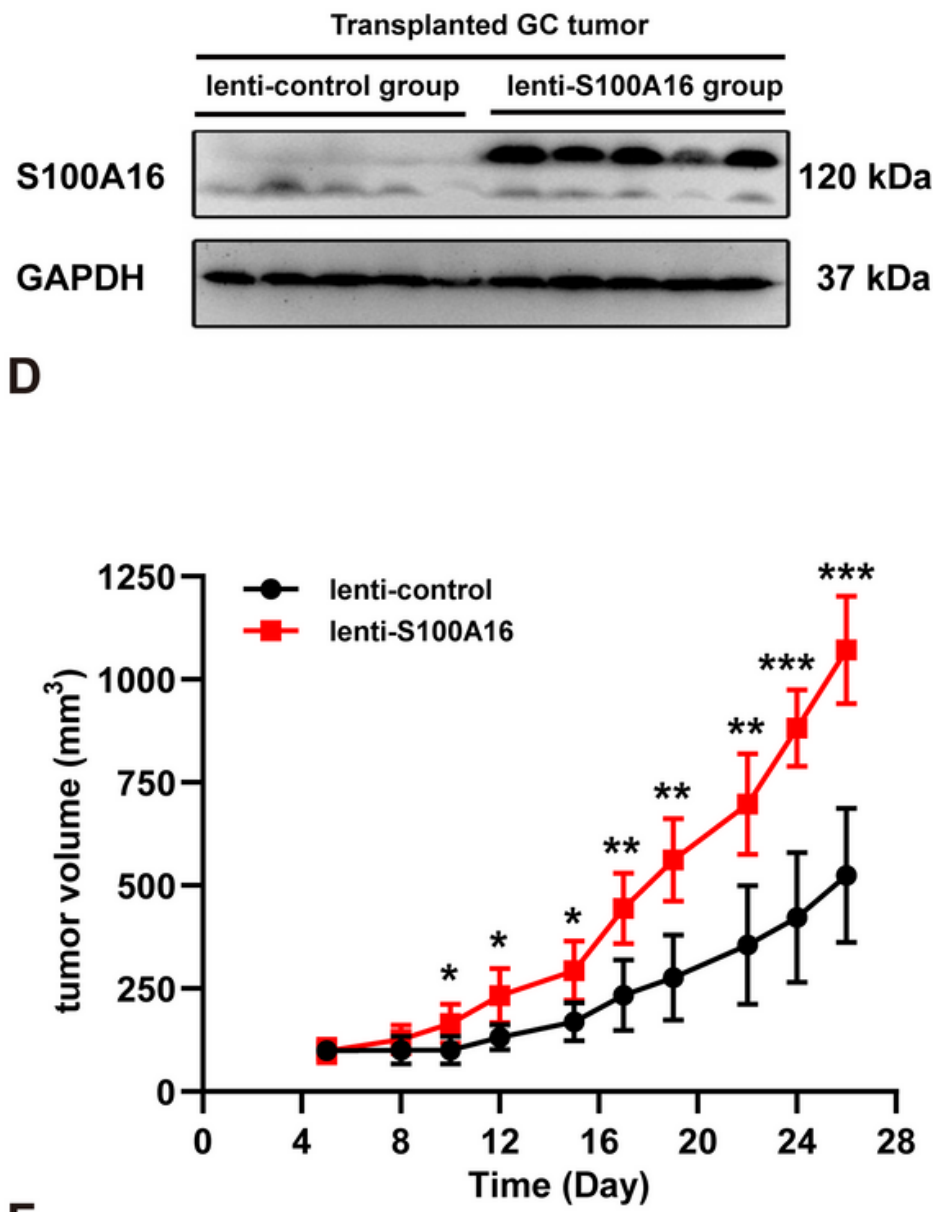

$\mathbf{F}$

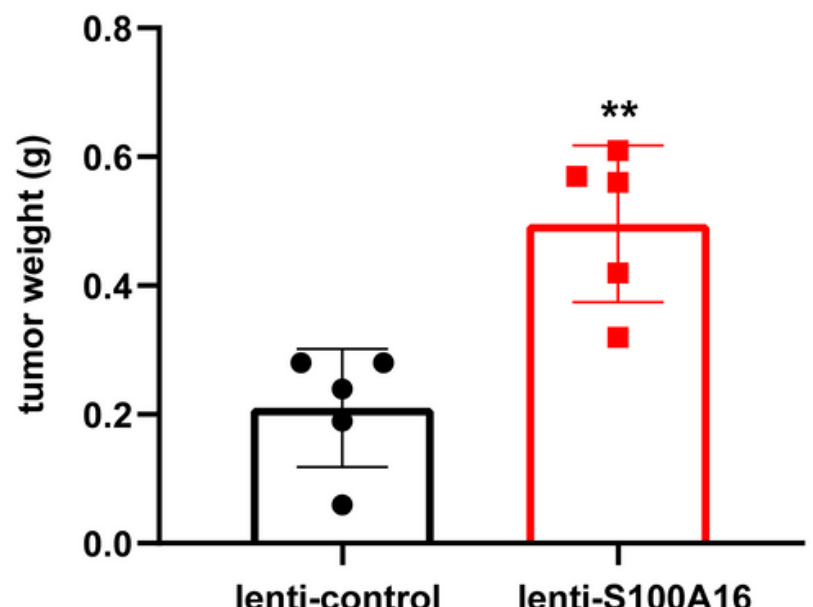

Figure 4

Overexpression of S100A16 enhances GC cells tumorigenesis in vivo. (A) Diagram of subcutaneous tumor transplantation in nude mice. (B-C) S100A16 overexpression were confirmed by Western blotting (B) and immunohistochemistry (C) in transplanted tumor tissues formed by SGC-7901 cells. Scare bar = $200 \mu \mathrm{m}$. (D) The volume for transplanted GC tumor was measured every 4-5 days since $5 \mathrm{~d}$ after injection. (E-F) The size and weight for xenograft tumors after sacrificed. Data are presented as mean \pm SD. $n=5$ for each group. For $D$ and $F, * P<0.05$, ** $P<0.01$, *** $P<0.001$ vs. lenti-control group. 
A

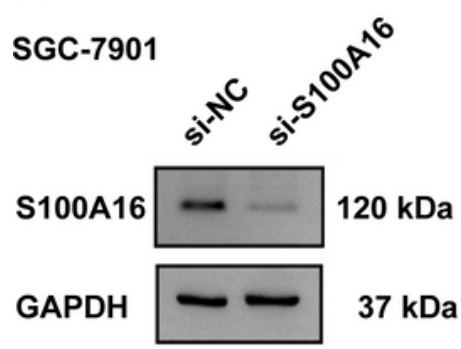

E

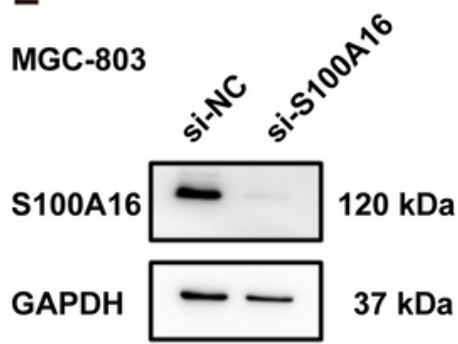

B

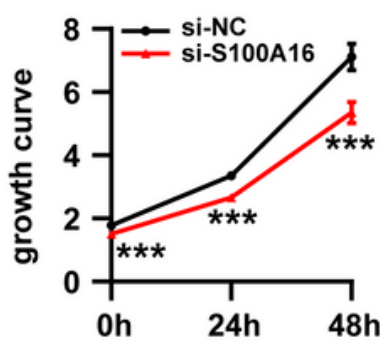

F
C

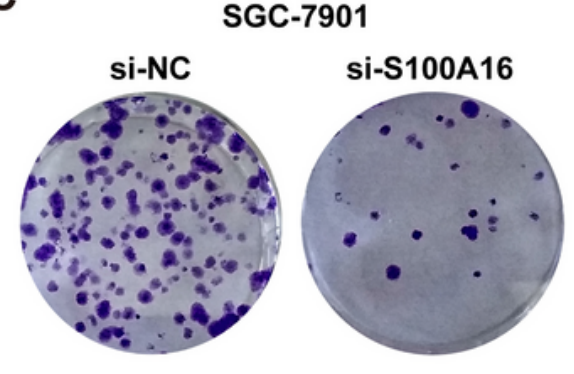

G

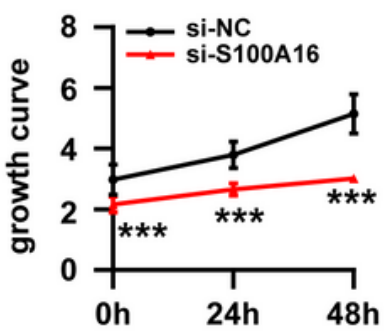

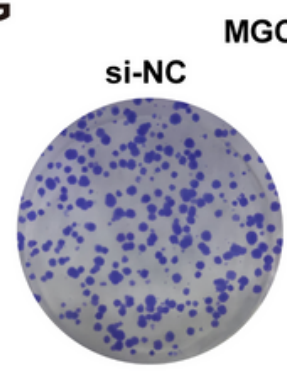

MGC-803

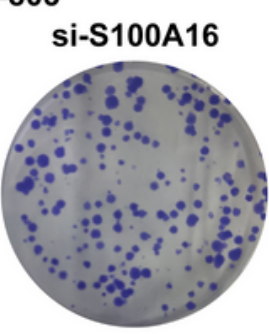

J

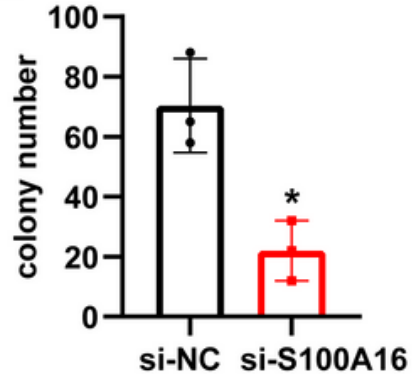

H

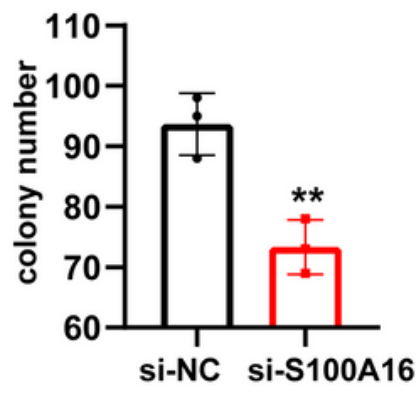

I

Si-NC

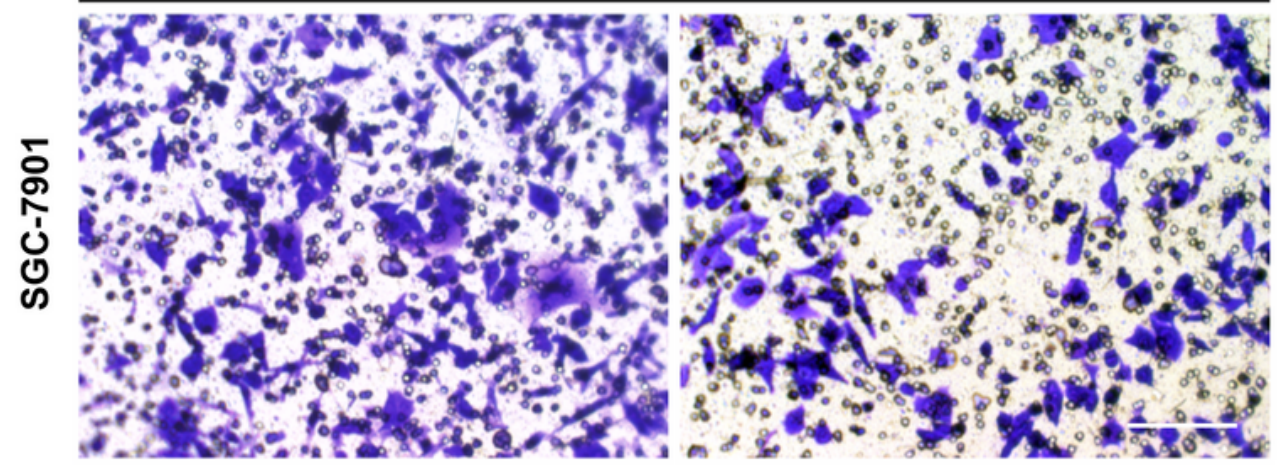

K

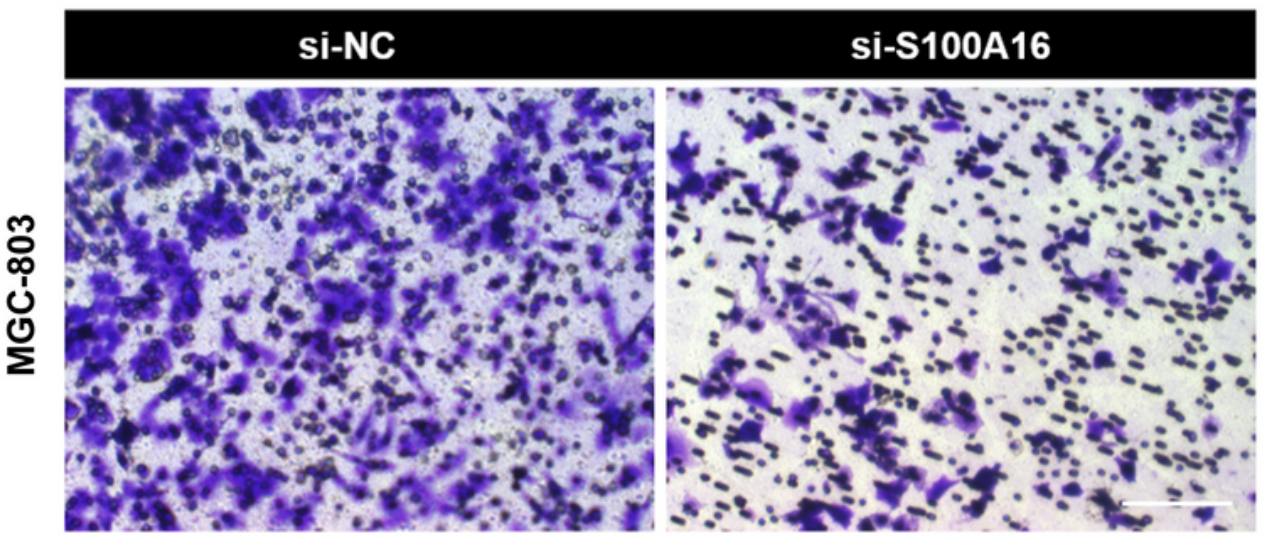

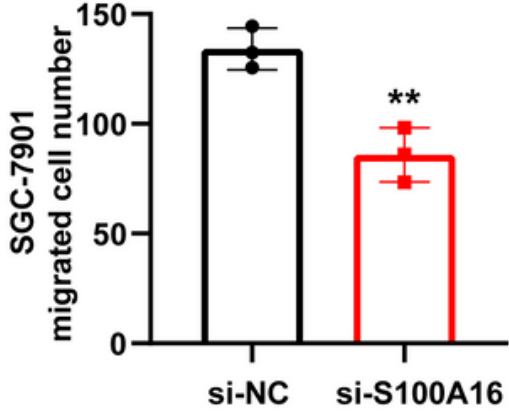

L

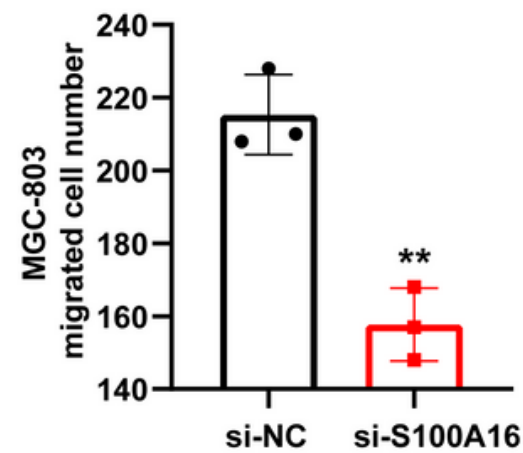

Figure 5

S100A16 inhibition suppresses GC cells growth and migration. (A) S100A16 knockdown in SGC-7901 cells were confirmed by Western Blotting. (B) Cell growth was measured by SRB staining in SGC-7901 cells transfected with si-NC and si-S100A16. (C) Colonies formed by SGC-7901 cells in (B). (D) Colonies number in (C) were quantified. (E) S100A16 knockdown in MGC-803 cells were confirmed by Western Blotting. (F) Cell growth was measured by SRB staining in MGC-803 cells transfected with si-NC and si- 
S100A16. (G) Colonies formed by MGC-803 cells in (F). (H) Colonies number in (G) were quantified. (I-J) The migration ability of SGC-7901 cells transfected with si-NC and si-S100A16 was measured by transwell assay. Migrated cell numbers were counted in (J). Scare bar $=100 \mu \mathrm{m}$. (K-L) The migration ability of MGC-803 cells transfected with si-NC and si-S100A16 was measured by transwell assay. Migrated cell numbers were counted in (L). Scare bar $=100 \mu \mathrm{m}$. Data are presented as mean $\pm S D . n=3$ for each group. For $B, D, F, H, J$ and $L$, * $P<0.05$, ** $P<0.01$, *** $P<0.001$ vs. si-NC group.

A

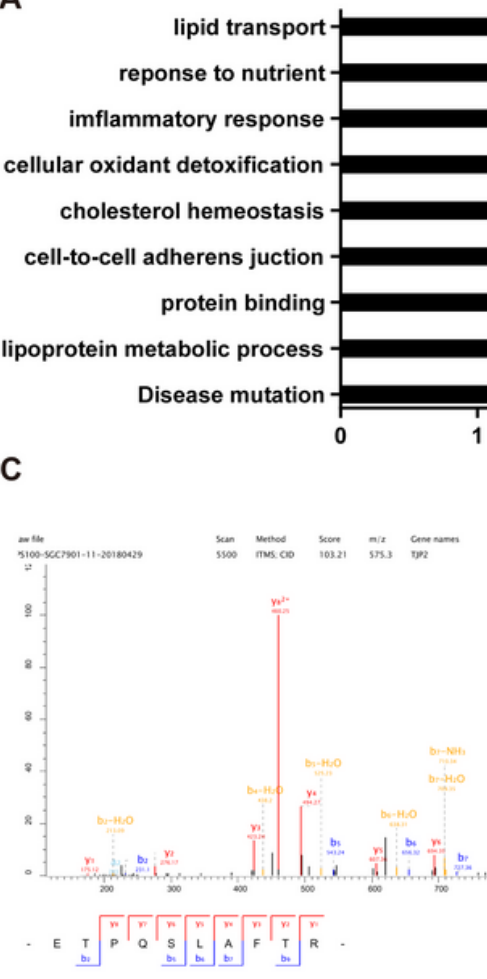

G

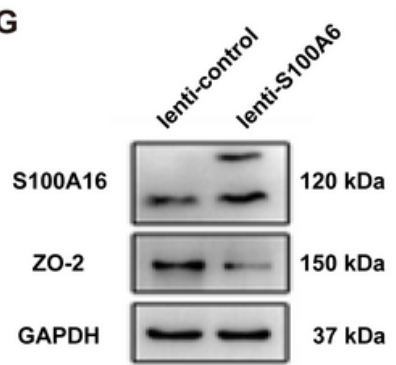

H

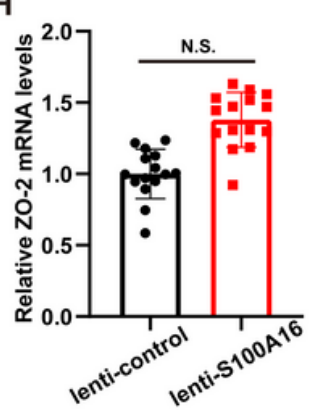

B

\begin{tabular}{|l|c|}
\hline \multicolumn{1}{|c|}{ Enriched GO term } & -log10 P value \\
\hline $\begin{array}{l}\text { Cell-to-cell adherens junction } \\
\text { Gene: TJP2 (ZO-2), JUP, HSP90AB1 }\end{array}$ & 3.27 \\
\hline
\end{tabular}

F

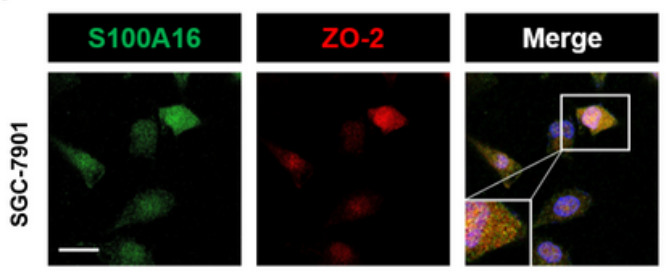

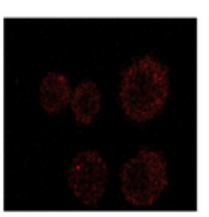

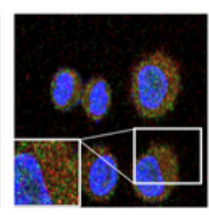

K

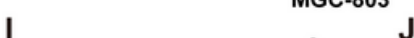

I
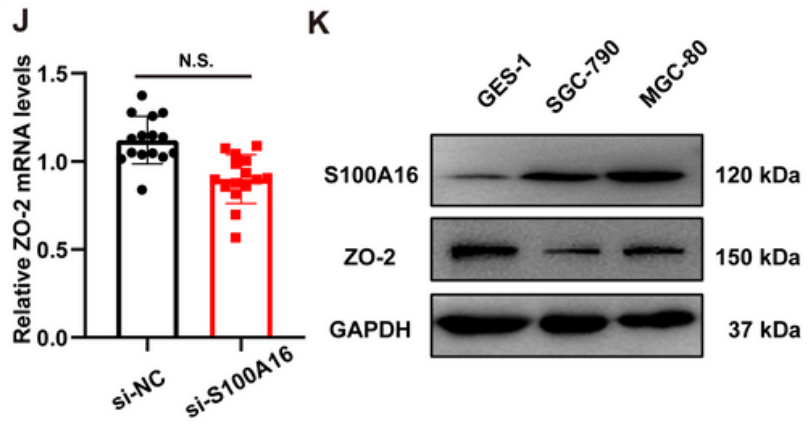

\section{Figure 6}

S100A16 and ZO-2 interact directly and their expression levels are inversely correlated in GC cells. (A) DAVID Bioinformatics Resources 6.7 was used to perform GO analysis of S100A16-binding proteins in SGC-7901 cells transfected with lenti-S100A16 or lenti-control (A). Relative cellular processes were sorted by $-\log (P$ value $)$. $P$ value < 0.05; $-\log (P$ value $)>1.3$. (B) A cluster of genes (ZO-2, JUP, HSP90AB1) associated with the cell-to cell adherens junction (B) was sorted out. (C) A digested peptide from ZO-2 in LC-MS/MS analysis of protein complex coimmunoprecipitated with S100A16 in SGC-7901 cells. (D-E) Validation of the interactions between endogenous S100A16 and Z0-2 in SGC-7901 (D) and MGC-803 cells (E). (F) ZO-2 co-localizes with S100A16 in gastric cancer cells. Immunofluorescence staining was 
performed for ZO-2 (red), S100A16 (green), and DAPI (blue). Scale bars $=10 \mu \mathrm{m}$. (G) The whole cell protein levels of S100A16 in SGC-7901 cells stably overexpressing S100A16 were measured through Western blot analysis. (H) ZO-2 mRNA level in SGC-7901 cells in G was detected via qRT-PCR assay. (I) The whole cell protein levels of S100A16 in SGC-7901 cells transfected with si-negative control (NC) or siS100A16 for $60 \mathrm{~h}$ were measured through Western blot analysis. (J) ZO-2 mRNA level in SGC-7901 cells in I was detected via qRT-PCR assay. (K) The expression of S100A16 and ZO-2 in GES-1, SGC-7901, and MGC-803 cells.

A

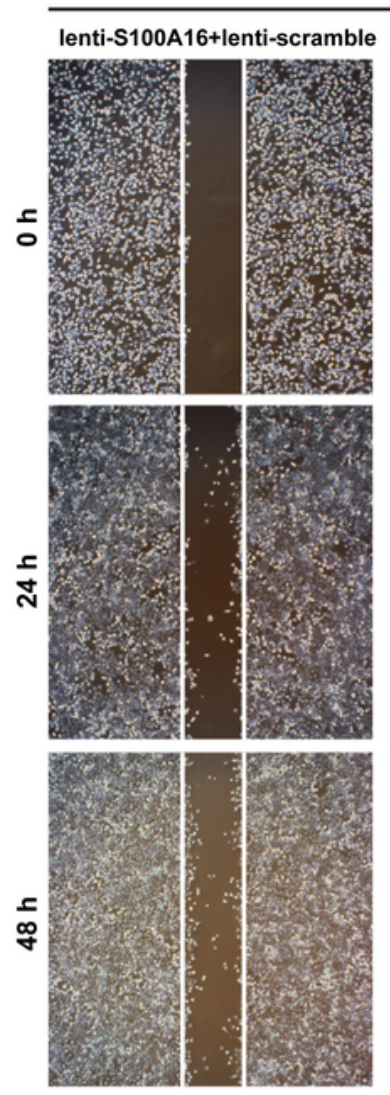

SGC-7901
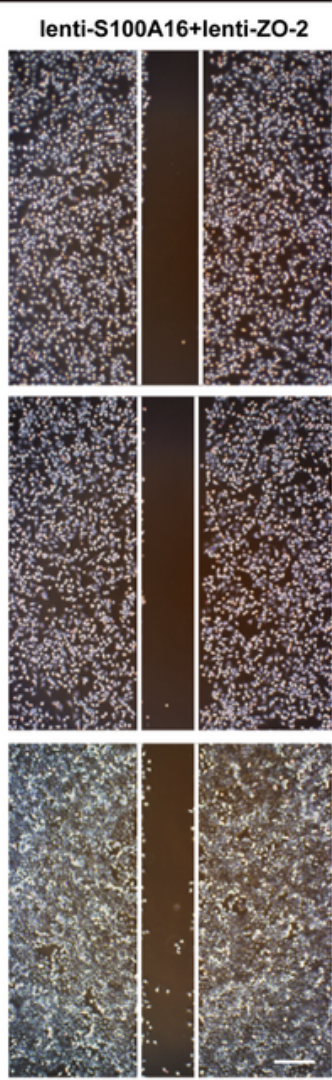

$\overbrace{}^{100}]^{\bullet}$ lenti-S100A16+lenti-scramble = lenti-S100A16+lenti-ZO-2
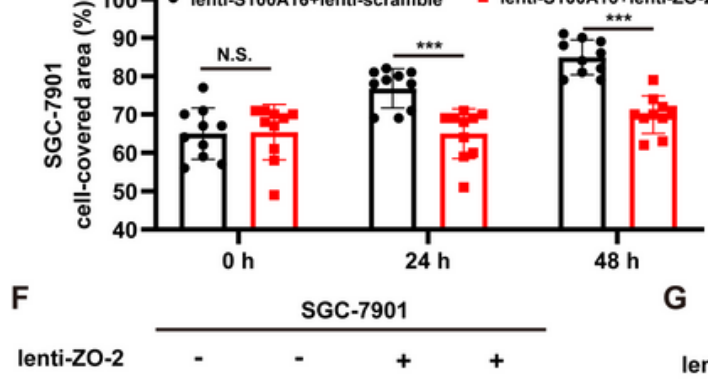

lenti-S100A16

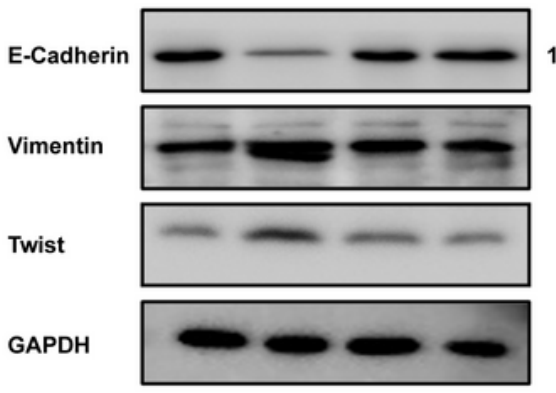

B
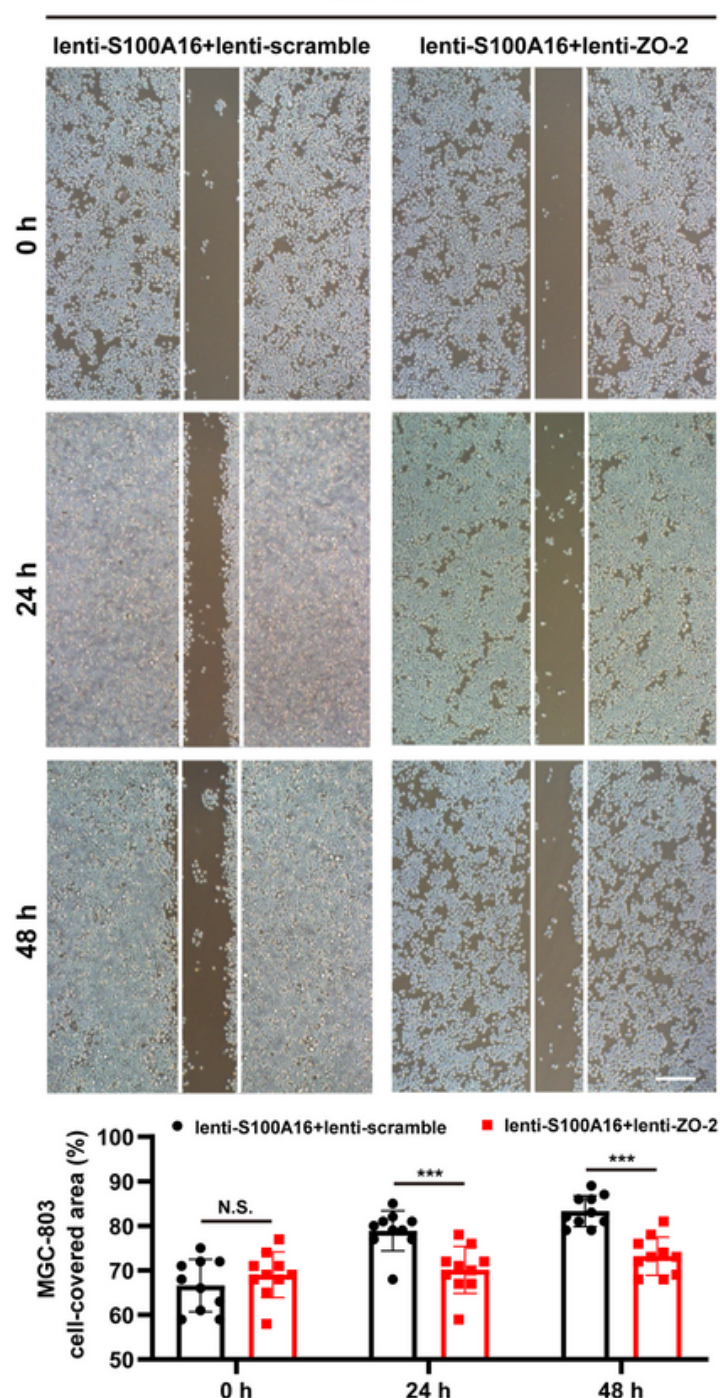

$\mathrm{H}$
C

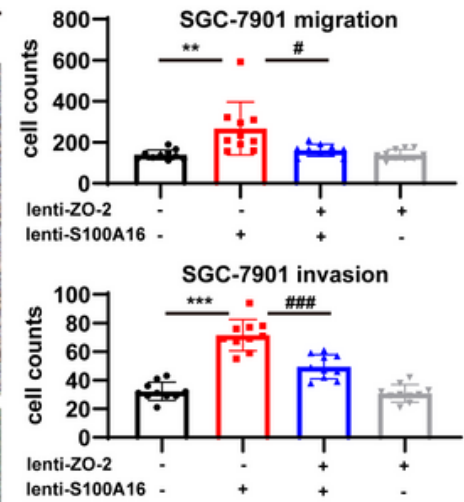

D

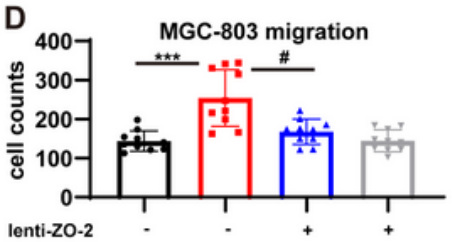

lenti-ZO-2 lenti-S100A16 .

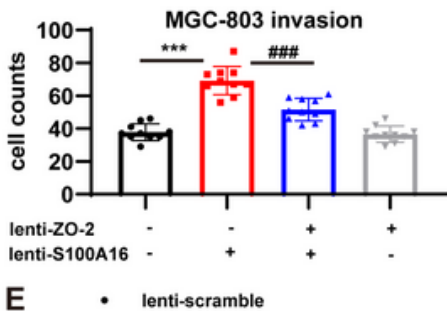

lenti-scramble lenti-S100A16 lenti-scramble+lenti ZO-2

हิ 4007 ienti-scramble+lenti Z

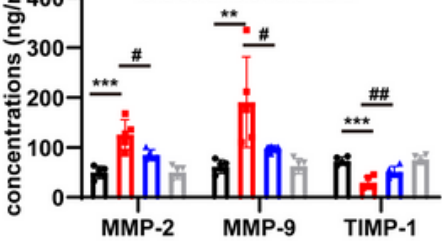

MGC-803

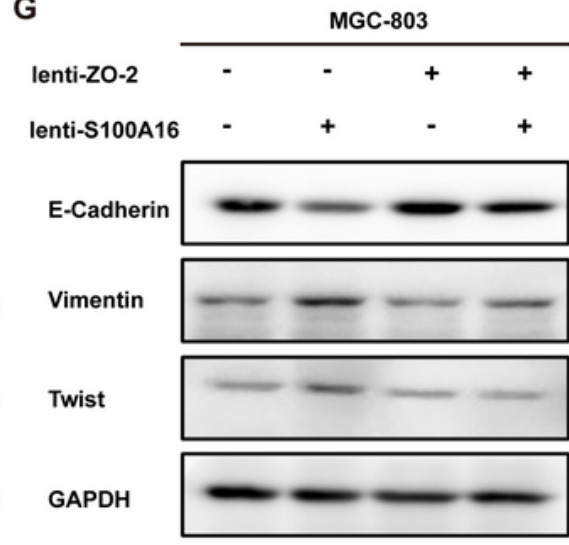

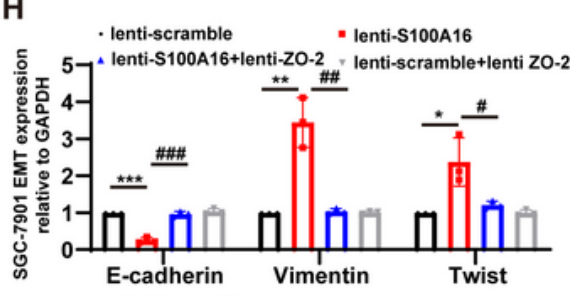

57 kDa - lenti-scramble "lenti-S100A16

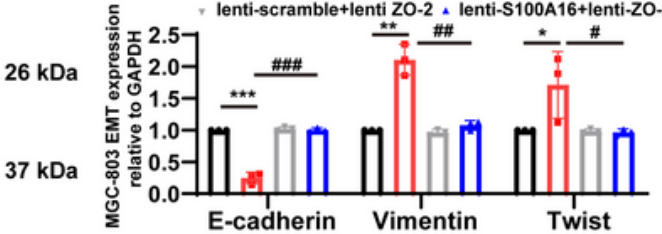




\section{Figure 7}

S100A16 triggers the process of invasion, migration and EMT in GC cells via ZO-2 inhibition. (A-B) The scratch wound-healing assays of SGC-7901 (A) and MGC-803 cells (B) stably overexpressing S100A16/ZO-2 or singly S100A16. Quantitative analysis was done below. Scare bar $=100 \mu \mathrm{m}$. (C-D) SGC7901 (C) and MGC-803 cells (D) migration and invasion were measured by transwell assays. Cells were quantified. (E) MMP-2, 9 and TIMP-1 levels in culture supernatant of SGC-7901 cells were measured by ELISA assays. (F-H) The protein levels of EMT markers (E-Cadherin, Vimentin and Twist) in SGC-7901 (F) and MGC-803 cells (G). GAPDH was used as internal standard. Grey density was quantified in H. Data are presented as mean \pm SD. $n=3-10$ for each group. For $A-B, * P<0.05$, $* * P<0.01, * \star * P<0.001$ vs. lentiS100A16+lenti-scramble group. For $C-E, H, * P<0.05$, ** $P<0.01$, *** $P<0.001$ vs. lenti-scramble group, \# $P<0.05$, \#\# $P<0.01$, \#\#\# $P<0.001$ vs. lenti-S100A16 group. 
A
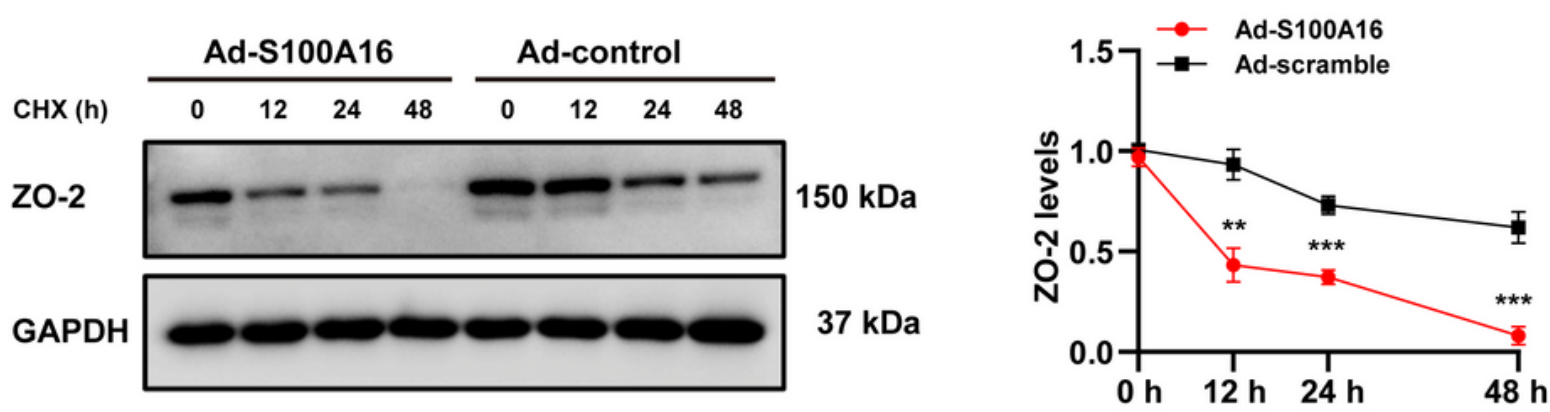

B

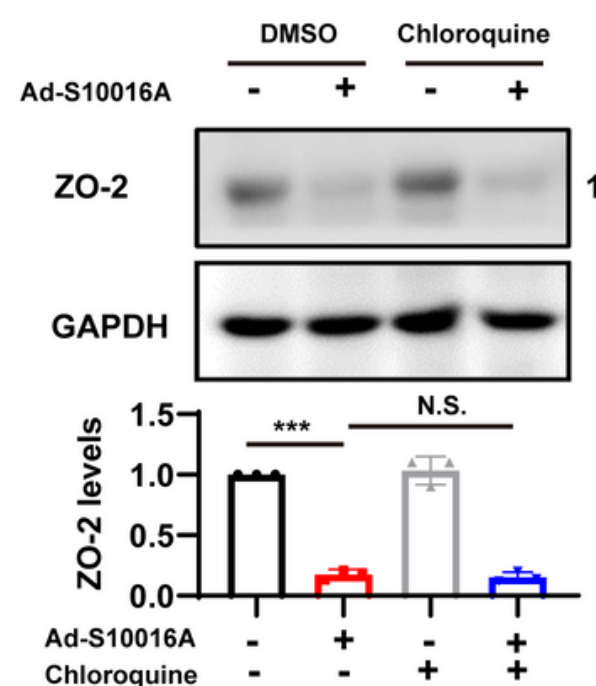

C
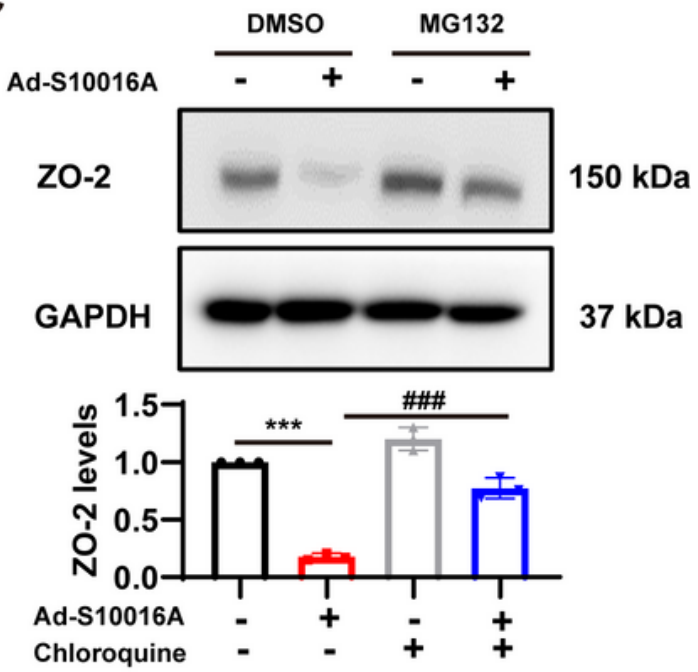

D

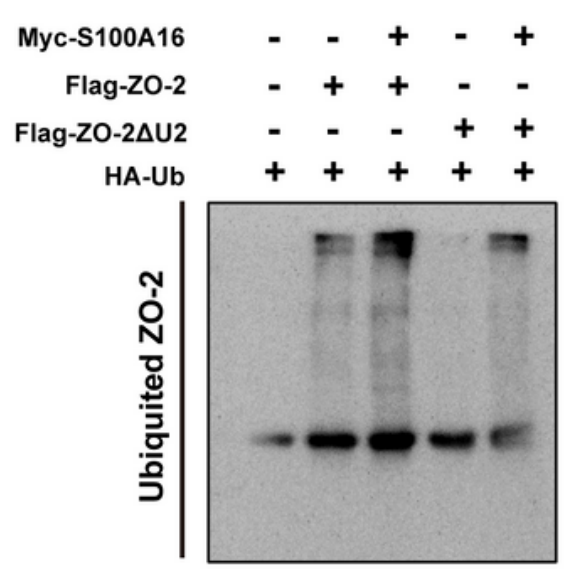

E

$150 \mathrm{kDa}$

$37 \mathrm{kDa}$

$37 \mathrm{kDa}$

Chloroquine

S100A16 ${ }^{\text {low }}$ GC cells $\quad$ S100A16 $6^{\text {high }}$ GC cells

Flag-ZO-2

Myc-S100A16

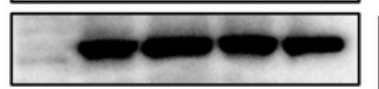

GAPDH
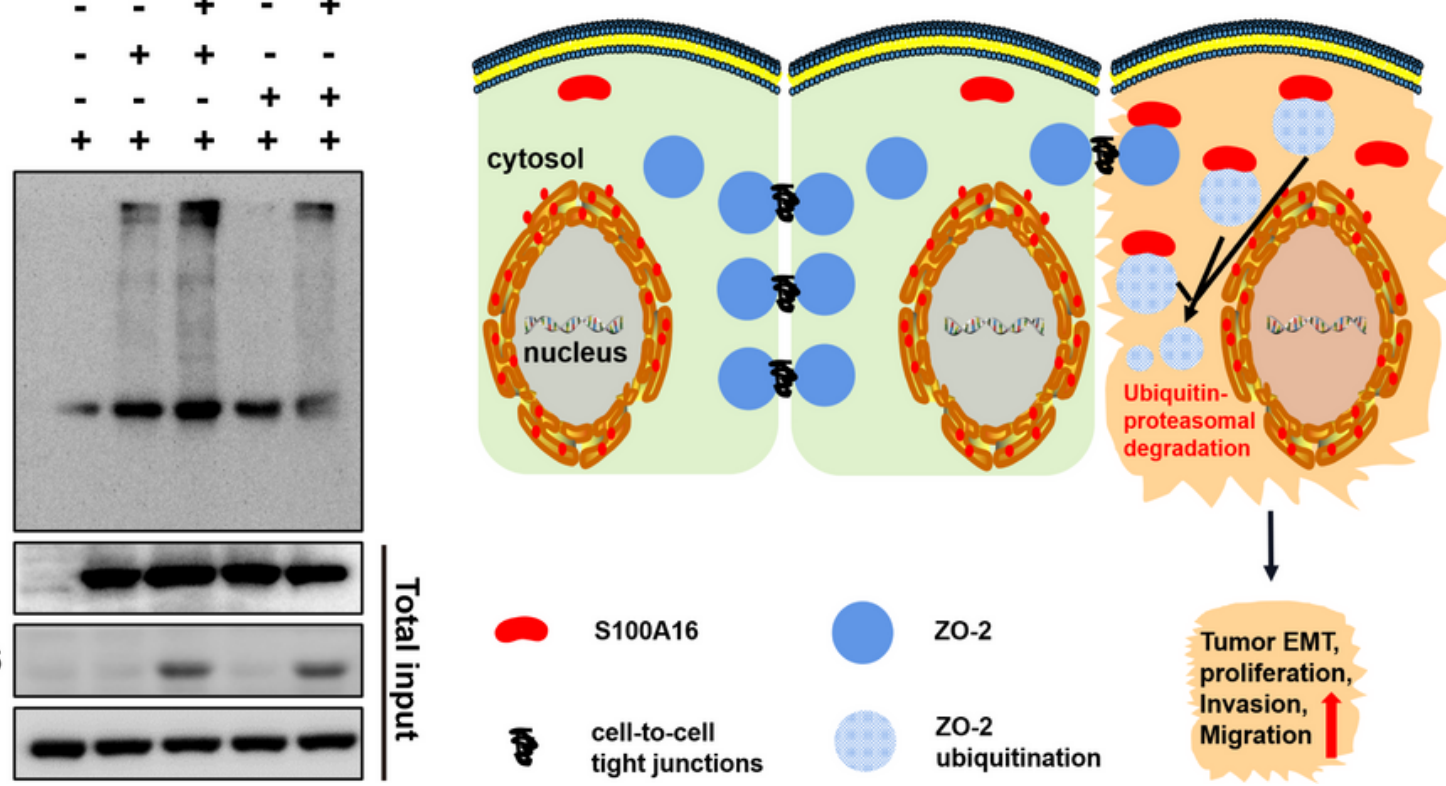

Figure 8

S100A16 contributes to ZO-2 ubiquitination and degradation. (A) SGC-7901 cells were transfected with Ad-S100A16 or Ad-control for $6 \mathrm{~h}$. After co-culture with $50 \mathrm{mmol} / \mathrm{I}$ cycloheximide (CHX) for $0,12,24$, or 48 $\mathrm{h}$, Western blot analysis was performed, and the relative ZO-2 expression was calculated on the right. (B) SGC-7901 cells were transfected with Ad-control or Ad-S100A16 for $6 \mathrm{~h}$ and then treated with or without chloroquine (50 $\mu \mathrm{mol} / \mathrm{I})$ for another $48 \mathrm{~h}$; ZO-2 expression was measured through Western blot analysis. 
(C) SGC-7901 cells were transfected with Ad-control or Ad-S100A16 for $6 \mathrm{~h}$ and then treated with or without MG132 $(10 \mu \mathrm{mol} / \mathrm{l})$ for another $48 \mathrm{~h}$; ZO-2 expression was measured through Western blot analysis. (D) SGC-7901 cells were co-transfected with Ub-HA, S100A16-Myc, and ZO-2-Flag or ZO-2- $\Delta$ U2 region-Flag expression plasmids for $18 \mathrm{~h} ; 6 \mathrm{~h}$ after treatment with proteasome inhibitor MG132 (10 $\mu \mathrm{mol} / \mathrm{l})$, the cells were lysed, and the supernatant was collected for an IP experiment. Flag-Tag antibody was used for immunoprecipitation, and HA-Tag antibody was used for Western blot analysis to measure the level of exogenous ZO-2 ubiquitination. (E) A proposed model of S100A16 mediating ZO-2 inhibition via ubiquitin-proteasomal degradation in GC metastasis. Data are presented as mean $\pm S D$. $n=3$ for each

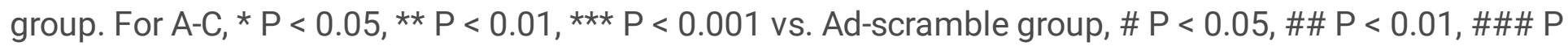
$<0.001$ vs. Ad-S100A16 group.

\section{Supplementary Files}

This is a list of supplementary files associated with this preprint. Click to download.

- Supplementarymaterials.docx 